\title{
1 Evolution of plasmid mobility: origin and fate of non-conjugative 2 plasmids
}

3 Charles Coluzzi ${ }^{1}$, Maria del Pilar Garcillán-Barcia ${ }^{2}$, Fernando de la Cruz ${ }^{2}$, Eduardo P.C. Rocha ${ }^{1}$

$4 \quad{ }^{1}$ Institut Pasteur, Université de Paris, CNRS, UMR3525, Microbial Evolutionary Genomics, Paris, 75015, France.

$5{ }^{2}$ Instituto de Biomedicina y Biotecnología de Cantabria (IBBTEC), Universidad de Cantabria-CSIC, C/Albert Einstein

622,39011 , Santander, Spain

7

\section{Abstract}

9 Conjugation drives horizontal gene transfer of many adaptive traits across prokaryotes. Yet, 10 only a fourth of the plasmids encode the functions necessary to conjugate autonomously, 11 others being non-mobile or mobilizable by other elements. How these different plasmids evolve 12 is poorly understood. Here, we studied plasmid evolution in terms of their gene repertoires and 13 relaxases. We observed that gene content in plasmid varies rapidly in relation to the rate of 14 evolution of relaxases, such that plasmids with 95\% identical relaxases have on average fewer 15 than $50 \%$ of homologs. The identification of 249 recent transitions in terms of mobility types 16 revealed that they are associated with even greater changes in gene repertoires, possibly 17 mediated by transposable elements that are more abundant in such plasmids. These changes include pseudogenization of the conjugation locus, exchange of replication initiators, and extensive gene loss. In some instances, the transition between mobility types also leads to the genesis of novel plasmid taxonomic units. Most of these transitions are short-lived, suggesting a source-sink dynamic, where conjugative plasmids constantly generate mobilizable and putatively non-mobilizable plasmids by gene deletion. Yet, in few cases such transitions resulted in the emergence of large clades of relaxases present only in mobilizable plasmids, suggesting successful specialization of these families in the hijacking of diverse conjugative systems. Our results shed further light on the huge plasticity of plasmids, suggest that many non-conjugative plasmids emerged recently from conjugative elements and allowed to quantify how changes in plasmid mobility shape the variation of their gene repertoires. 
Bacteria acquire DNA from other cells, eventually of different species, by multiple mechanisms of horizontal gene transfer (HGT). These genes can be integrated into the genome and expressed, thus providing adaptive phenotypic shifts (de la Cruz, Davies 2000; Soucy et al. 2015). In many species, HGT is in large part, or entirely, driven by the transfer of mobile genetic elements (MGEs) carrying accessory genes of adaptive value (Rankin et al. 2011; Hall et al. 2017; Partridge et al. 2018). Some of these, plasmids and Integrative Conjugative Elements (ICE), encode a mating pair formation (MPF) machinery that includes a type 4 secretion system (T4SS) which transfers one DNA strand from the donor to the recipient cell (de la Cruz et al. 2010). In plasmids, conjugation starts by the action of a relaxase, a multi-domain protein with transesterification activity, that nicks the DNA molecule at the origin of transfer (oriT) and links covalently to a single strand (Gonzalez-Perez et al. 2007). The nucleoprotein filament is then presented to the T4SS by a type 4 coupling protein and transferred to the other cell where it is circularized. Finally, the replication machineries of donor and recipient cells restore the double strand of the plasmids. The amounts of contiguous DNA that can be transferred by conjugation are without equal among the mechanisms of HGT, since entire chromosomes can be transferred in one single event (Adelberg, Pittard 1965). One other specificity of conjugation is to allow transfer across bacteria that are distantly related.

Both ICEs and conjugative plasmids conjugate MGEs that are not capable of autonomous conjugation, so called mobilizable plasmids and integrative mobilizable elements (reviewed in (Ramsay, Firth 2017)). Mobilization in trans occurs when the element uses a conjugative system encoded by another element for horizontal transfer. The best studied cases concern plasmids lacking a functional conjugation system and encoding an oriT and a relaxase. The relaxase interacts with the oriT and then with the T4SS encoded by another element, which is used for plasmid transfer. Here, plasmids are called conjugative when they encode a presumably complete machinery for conjugation (relaxase and MPF) and are called mobilizable when they encode a relaxase gene. It has often been observed that rates of conjugation of mobilizable plasmids are lower than those of the co-occurring conjugative plasmid (Perez-Mendoza et al. 2006 ; Blanca-Ordóñez et al. 2010; Klümper et al. 2014). Other MGEs, plasmids or elements integrated into the chromosome, only have an oriT (O'Brien et al. 2015; Yui Eto et al. 2021). In this case, the oriT interacts with a relaxase encoded in trans that then presents the nucleoprotein to its cognate T4SS. Since such plasmids only encode oriT for their mobility, and these sequences are more difficult to identify using bioinformatic tools, such plasmids are hard

61 to class in terms of mobility using computational approaches. We will refer to plasmids lacking

62 relaxases as pMOBless. They include all plasmids that do not encode a relaxase and for which, 63 therefore, we ignore if they can be mobilized by conjugation. Work done a decade ago has 64 shown that many completely sequenced genomes have plasmids and, among these plasmids, a 
similar number of conjugative and mobilizable plasmids is found (Smillie et al. 2010). Mobilizable plasmids tend to be smaller than conjugative plasmids, presumably because they do not need to encode the large locus associated with the MPF.

Phylogenetic and functional studies have revealed that conjugative plasmids and ICEs are very similar from the point of view of their conjugation machinery and interconversions between the two are frequent (Guglielmini et al. 2011; Johnson, Grossman 2015; Cury et al. 2018). There are eight large groups of MPF, two are specific to monoderms, incl. Archaea, and the remaining are found in diderms (Guglielmini et al. 2013). There are 9 well-known classes of relaxases that vary in their sequence, protein domains and sometimes in how they catalyze chemical reactions (Garcillan-Barcia et al. 2020), and novel putative ones are being unraveled by computational studies (Coluzzi et al. 2017). The relaxase (that defines the MOB class) is one of the few key taxonomic traits of plasmids (Garcillan-Barcia et al. 2009). Recently, the analysis of average nucleotide identity (ANI) between plasmids revealed that closely-related plasmids cluster in socalled plasmid taxonomic units (PTUs) (Redondo-Salvo et al. 2020). PTUs regroup plasmids sharing more than half of their genomes, tend to have one single type of relaxase and have a characteristic host distribution, most frequently with host ranges beyond the species barrier.

Conjugative and mobilizable elements transfer many kinds of traits, including virulence factors and symbiotic islands (Nuti et al. 1979 ; Johnson, Nolan 2009 ; Carattoli 2013). Their ability to transfer large amounts of DNA per event allows the spread of complex traits encoded in long genetic loci (Kobayashi 2018; Geng et al. 2021). The broad host range of conjugation allows transfer across bacterial Phyla and beyond, e.g. between Proteobacteria and Firmicutes (TrieuCuot et al. 1987) or from Proteobacteria to plants (Lacroix, Citovsky 2018). This trait may explain why conjugative elements drive the transfer of antibiotic resistance genes from distantly related bacteria to nosocomial pathogens (Pedersen et al. 2018; Che et al. 2021). But conjugation is also costly because it requires production, assembly and functioning of a large protein complex that spans multiple membranes and renders bacteria sensitive to certain phages (San Millan, Craig MacLean 2019). In this context, the added cost of having a mobilizable plasmid might be low, since the conjugative apparatus is encoded by the conjugative element. Hence, relative to conjugative elements, mobilizable elements depend on an MPF encoded in trans but may be less costly to the cell. The dependency of these plasmids on other conjugative elements can have some advantages. Some relaxases can be mobilized by different conjugative systems in trans (Garcillan-Barcia et al. 2019), allowing mobilizable plasmids to increase their frequency of transfer. Hence, traits encoded in conjugative or mobilizable elements may enjoy different patterns of genetic mobility. Such traits may also transit from one type of plasmid to the other, since there is interconversions or gene flow between conjugative and mobilizable elements (Liu et al. 2013). This can be due to translocations or fusion between replicons, scissions of plasmids, or gene deletions. To understand the evolution of conjugative elements 
102 and their impact on bacterial genomes, one thus needs to understand the evolutionary

103 interplay between conjugative and mobilizable elements.

104 While the macro-evolution of the machinery for conjugation, the MPF, has been described in 105 detail (Guglielmini et al. 2013), we know relatively little of the general patterns of evolution of 106 plasmids relative to their categorization as mobilizable or conjugative. To shed light on this 107 issue, we analysed a large set of plasmids in terms of their mobility by conjugation. Plasmids are 108 better suited for such studies than ICEs, because they are better known and easier to identify 109 accurately in complete genome sequences. To understand the evolution of conjugative and 110 mobilizable plasmids, we assessed the relative frequency of each relaxase in mobilizable and 111 conjugative plasmids and used the relaxases as phylogenetic markers to trace past events of 112 change. These analyses revealed a source-sink dynamics where conjugative plasmids frequently 113 produce mobilizable and MOBless elements. These transitions are associated with major 114 disruptions of gene repertoires and shed light on how mobilizable and MOBless plasmids are 115 formed. They also suggest that relaxases endure different selective pressures in conjugative 116 and mobilizable elements. 


\section{Plasmid Dataset}

120 The dataset used in this study consist of 13,525 complete genomes from 2,421 species

121 retrieved from NCBI RefSeq database of high quality complete non-redundant prokaryotic 122 genomes (ftp://ftp.ncbi.nlm.nih.gov/genomes/refseq/, last accessed in May 2019). These

123 genomes contain 11,805 plasmids. To avoid the misidentification of ICEs as conjugative

124 plasmids in chromids or secondary chromosomes, we excluded from further study the 419

125 plasmids larger than $500 \mathrm{~kb}$ (Harrison et al. 2010; Smillie et al. 2010). The complete list of

12611,386 plasmids can be found in Table S1.

127 Detection of conjugative systems

128 The detection of conjugative systems was performed using MacSyFinder v.1.0.5 (Abby et al. 2014). Briefly, MacSyFinder uses HMM protein profiles and a set of rules (which are defined in MPF model files) about their presence and genetic organization to identify occurrences of given MPFs and relaxases in a genome. MacSyFinder was used with default parameters (HMMer evalue $<0.001$, HMM profile alignment coverage $>50 \%$ ) and was ran independently for each MPF type. To detect conjugative systems in bacterial chromosomes, we used protein HMM profiles and the MPF system definitions as described before (Cury et al. 2017).

To detect conjugative systems in plasmids, we changed the default procedure to allow for relaxase genes that are distant from the genes encoding the MPF. Hence, relaxases and T4SS were searched independently. We searched for relaxases using the same protein profiles with HMMer v.3.2.1 (e-value < 0.001, HMM profile alignment coverage > 50\%)(Eddy 2011). The T4SS were searched using MacSyFinder with modified MPF system definitions: the modified definitions allow the relaxase to be absent and the MPF proteins to be at any distance from each other's in the replicon (contrary to the $60 \mathrm{~kb}$ of space for the chromosome models). Replicons encoding both a relaxase and a complete MPF were considered conjugative.

143 Recently, a novel family of relaxases, $\mathrm{MOB}_{\mathrm{L}}$, was identified in Firmicutes (Ramachandran et al.

144 2017). We made a protein profile for $M_{O} B_{L}$ with HMMER v.3.2.1 using the 817 proteins 145 sequences described as $\mathrm{MOB}_{\llcorner}$homologs in (Ramachandran et al. 2017). The search for elements of this family of relaxases revealed an important overlap with relaxases detected with the $\mathrm{MOB}_{\mathrm{P} 2} \mathrm{HMM}$ profile, in agreement with previous works (Garcillan-Barcia et al. 2020), and 
151 A plasmid was considered conjugative ( $\mathrm{pCONJ}$ ) when it encoded one or several relaxases, a 152 VirB4, a T4CP, and a minimum number of additional MPF proteins. The latter threshold varies 153 according to the MPF type: two proteins for MPFFA $_{F}$ and MPFFATA and three for the other MPF 154 types (B, C, F, G, I and T). These are values lower than previously used in (Cury et al. 2018) to make the assessment of a transition from pCONJ to PMOB conservative. A plasmid was

156

157

158

159

160

161

162

163

164

165

166

167

168

169

170

171

172

173

174

175

176

177

178

179

180

181

182 considered mobilizable ( $\mathrm{pMOB}$ ) when it encoded a relaxase but lacked the conditions to be conjugative (misses T4CP, VirB4 or a sufficient number of the other components). Plasmids encoding more than 5 MPF proteins tend to be predicted as pCONJ and plasmids encoding 5 or less MPF proteins tend to be predicted as PMOB (Figure S1). To pinpoint cases where plasmids encode several genes typical of conjugative elements, but not enough to allow classing them as pCONJ, we classed mobilizable plasmids containing in addition to the relaxase, more than 5 other proteins involved in the conjugative transfer (either MPF proteins, T4CP or additional relaxases) as decayed conjugative elements (pdCONJ).

\section{Relaxase clustering, alignments and phylogeny}

We clustered the 5,666 relaxase protein sequences using MMSeqs2 cluster (v. 9-d36de) with 99\% identity and alignment coverage (on both query and target) thresholds (i.e. parameters -min-seq-id 0.99 --cov-mode 0 -c 0.99) (Hauser et al. 2016). The protein sequences of the relaxases of each type were then aligned using MAFFT v.7.245 with the E-INS-I algorithm (parameters --genafpair --maxiterate 1000) (Katoh, Standley 2014). The multiple alignments were trimmed using ClipKIT with the 'kpic-gappy' algorithm which keeps only parsimony informative and constant sites and removed all sites above $90 \%$ gappyness (Steenwyk et al. 2020). The phylogenetic analyses were performed on the resulting multiple alignment using IQTREE (v.1.5.5, (Nguyen et al. 2015)) with the ultra-fast bootstrap option (1000 bootstraps) and with the best fitting model estimated ModelFinder Plus (-MPF) for each class of relaxases according to the BIC criterion. Trees were rooted using the midpoint function from the phangorn packages (v.2.5.5) for R.

\section{HMM-HMM alignments}

MOB protein alignments were retrieved from (Guglielmini et al. 2011) and then transformed into HMM profiles (with '-add cons' and '-M 50' parameters) using hhmake from HH-suite3.0 program v.3.3.0 (Steinegger et al. 2019). The alignment of HMM profiles was performed using hhalign from the same suite of programs with default parameters. We took into consideration only bi-directional alignments with a probability superior to $70 \%$ and an e-value inferior to $10^{-4}$. 
184 To identify plasmid replicons, we used PlasmidFinder 2.0.1 with the 2020-07-13 database and default parameters (Carattoli et al. 2014). We identified 200 replicon groups in 4827 of the 11386 plasmids.

wGRR estimation

188

189

190

191

192

193

We searched for significant similarity (e-value $<10^{-4}$, identity $\geq 35 \%$, coverage $\geq 50 \%$ ) among all pairs of plasmid proteins using MMseqs2 (v. 9-d36de). The best bi-directional hits (BBH) between pairs of elements were then used to calculate the weighted gene repertoire relatedness (wGRR) (Cury et al. 2018):

$$
w \operatorname{GRR}(A, B)=\frac{\sum_{i}^{P} i d\left(A_{i}, B_{i}\right)}{\min (\# A, \# B)}
$$

Where $A i$ and $B i$ are the $i$ th $B B H$ pair of $\mathrm{P}$ total pairs, $i d\left(A_{i}, B_{i}\right)$ is the identity between the $\mathrm{BBH}$ pair, and $\min (\# A, \# B)$ is the number of genes encoded by the plasmid encoding fewer genes. The WGRR varies between 0 and 1 . A wGRR close to 1 means that both plasmids are highly related (all genes in an element have a very similar BBH in the other) and a wGRR of 0 means that plasmids lack homologs.

\section{PTU predictions}

The taxonomic classifier of plasmids, COPLA, was used to assign plasmids to taxonomic units (Redondo-Salvo et al. 2021).

\section{Average Nucleotide Identity (ANI) calculation}

Pairwise ANI scores were obtained by splitting every genome into fragments of $1.0 \mathrm{~kb}$ using an sliding window algorithm with a step of $200 \mathrm{bp}$. The resulting sets of fragments were compared, all against all, with a $70 \%$ identity threshold over $70 \%$ of the fragment length. Reciprocal Best Hits were selected and, if the number of selected fragments covered at least $50 \%$ of the smaller plasmid, an ANI score was assigned. The network layout was obtained using Gephi (Bastian et al. 2009).

\section{Plasmid ORFeome network analysis}

We used ACCNET v 1.2to build the plasmid ORFeome network (Lanza et al. 2017). Homologous protein clusters were generated using kClust (v. 1.0) (Hauser et al. 2013), with $>95 \%$ protein identity, $>80 \%$ alignment coverage and clustering E-value $<10^{-14}$. All edges were assigned equal weights. The network layout was obtained using Gephi (Bastian et al. 2009). 
214 We inferred the ancestral state of each plasmid mobility type (pCONJ, pMOB and pdCONJ) with

215 PastML (v1.9.33) (Ishikawa et al. 2019). We used the maximum-likelihood algorithm MPPA

216 (marginal posterior probabilities approximation) and the F81 model, as recommended by the

217 authors. The MPPA algorithm chooses for every node a subset of ancestral states that

218 minimizes the prediction error measured by the Brier score. Hence, it may keep multiple state

219 predictions per node but only when they have similar and high probabilities. To avoid over-

220 estimation of the transitions between states, we counted transitions only when the ancestral

221 node had a unique state that was different from the derived state. This may result in a

222 conservative estimate of the total number of transitions. Out of the 287 observed transitions

223 for all trees combined, 38 were removed because of this stringent criterion, leaving 249

224 transitions to be analyzed.

225 Cumulative probability of transitions

226 For each tree, we computed the patristic distance matrix using the cophenetic.phylo function

227 from the package ape (v5.3) for R (Paradis, Schliep 2019). Based on the patristic distance

228 matrices, we measured the patristic distance separating each relaxase in our dataset from the

229 closest relaxase in the phylogenetic tree that was associated with a different mobility type. For

230 relaxases not present in the tree, i.e. not a representative of a cluster, we used the patristic

231 distance separating the representative of the cluster and the closest relaxase in the tree having

232 a different mobility type. This provides for each plasmid a patristic distance to the closest

233 plasmid of a different type. The empirical cumulative distribution function of these patristic

234 distances was computed for each MOB family and each mobility type, using the ECDF built-in

235 function for python (v3.6.10). Empirical cumulative distribution plots were computed using, the

236 ecdfplot function from the seaborn package (v0.11.0) for python (Waskom 2021).

237 Mobile plasmid-pMOBless associations

238 We paired each mobile plasmid with the MOBless plasmid with the highest wGRR. To avoid the 239 over-representation of the same transition event, if a MOBless plasmid was paired that way 240 with multiple mobile plasmids, we only kept the mobile/MOBless pair with the highest wGRR 241 value.

242 Statistics

243 Unless mentioned otherwise all statistics were performed within R (v3.6.2). Statistics between 244 two variables were done using standard non-parametric tests (Wilcoxon test). Chi-tests were 245 performed using the R (v3.6.2) chisq-test built-in function. 
Co-occurrence and wide distribution of conjugative and mobilizable plasmids

248

249

250

251

252

253

254

255

256

257

258

259

260

261

262

263

264

265

266

267

268

269

270

271

272

273

274

275

We identified relaxases and conjugative systems among 11,386 plasmids smaller than $500 \mathrm{~kb}$ (to avoid mis-assigned secondary chromosomes) from the completely assembled genomes of RefSeq (Table S1). Plasmids were classed in four classes (Figure 1A): 53\% of the plasmids lacked relaxases (pMOBless), 23.2\% were classed as conjugative (pCONJ) because they encode a relaxase and a sufficient number of MPF genes, $2.8 \%$ were classed as pdCONJ because they encoded more than 5 MPF genes but lacked the quorum of genes required to be conjugative, and $23.8 \%$ were classed as PMOB because they encoded a relaxase and 5 or less MPF genes. Hence, mobilizable and conjugative plasmids are present at similar frequencies and make up about half of all plasmids, consistent with previous results with smaller datasets (Smillie et al. 2010). The average size of plasmids varies with mobility type, with pCONJ (median size of $111.7 \mathrm{~kb}$ ) being slightly, but significantly, larger than pdCONJs (96kb) and the latter being much larger than pMOBs (37kb, $\mathrm{P}<0.001$, Wilcoxon tests, Figure S7). Many plasmids encode Insertion Sequences (IS) that are known to promote genetic variation. We identified ISs in all plasmids using ISEscan v1.7.2.2 default options and found the highest densities in pdCONJ and the lowest in MOBless plasmids (Figure 1B).

Our dataset of 5,666 relaxases includes all the different MOB classes, but with very different abundance (Figure $1 \mathrm{C}$ ). By far, the most prevalent classes were $\mathrm{MOB}_{\mathrm{F}}$ and $\mathrm{MOB}_{\mathrm{P}}$ which account for more than $62 \%$ of all relaxases, and are present in both pCONJs and pMOBs. It is important to note that the relative frequency of conjugative and mobilizable plasmids depends on the relaxase family $\left(\chi^{2}=1680.6, \mathrm{df}=18, \mathrm{P}<0.001\right)$. For example, $95 \%$ of the $\mathrm{MOB}_{\mathrm{V}}$ relaxases and $99 \%$ of those identified by the MOBP2 HMM profile are in mobilizable plasmids (pMOBs or pdCONJs) (Figure 1C). In contrast, $94 \%$ of $\mathrm{MOB}_{H}$ relaxases are in pCONJs. The average size of plasmids also varies in function of the relaxase family. Plasmids with $\mathrm{MOB}_{H}$ or $\mathrm{MOB}_{\mathrm{F}}$ relaxases tend to be larger, whereas those with $\mathrm{MOB}_{0}, \mathrm{MOB}_{T}$ and $\mathrm{MOB}_{v}$ are significantly smaller (Figure 1D). The trends across these types of relaxases are similar with pCONJ being in general the largest, closely followed by pdCONJ, whereas $\mathrm{pMOB}$ are smaller. Of note, pdCONJs to pCONJs are more similar in terms of relaxase family and plasmid size than they are of pMOB, suggesting that they were recently derived from pCONJ by gene loss. 

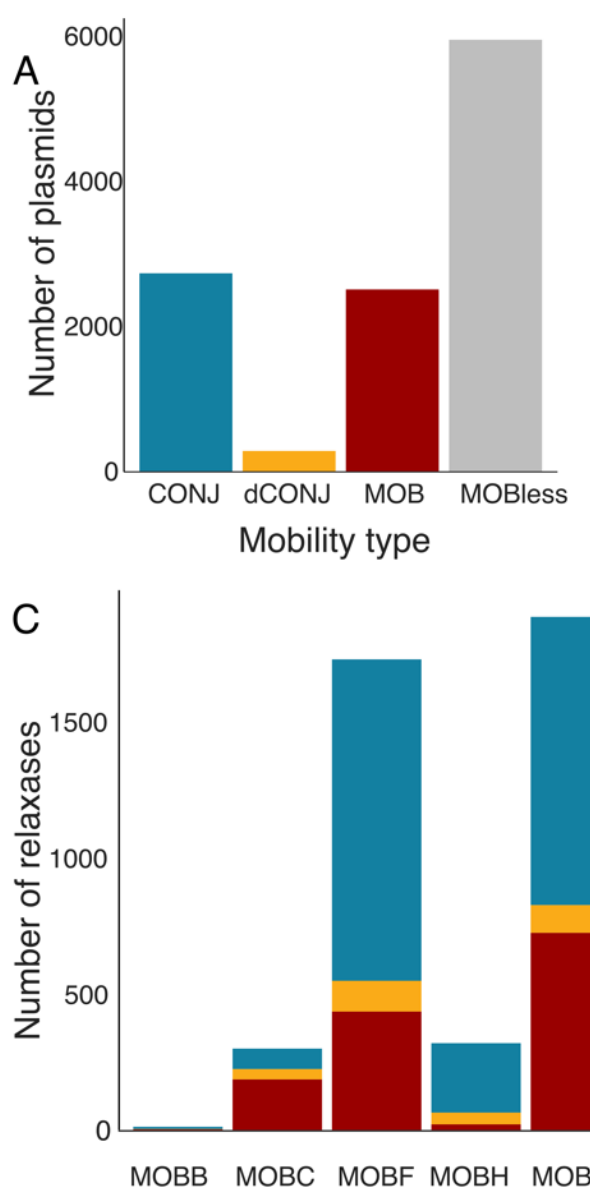

B

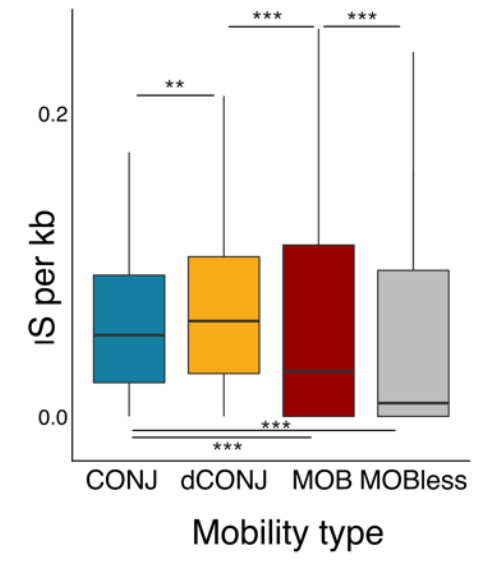

Mobility type
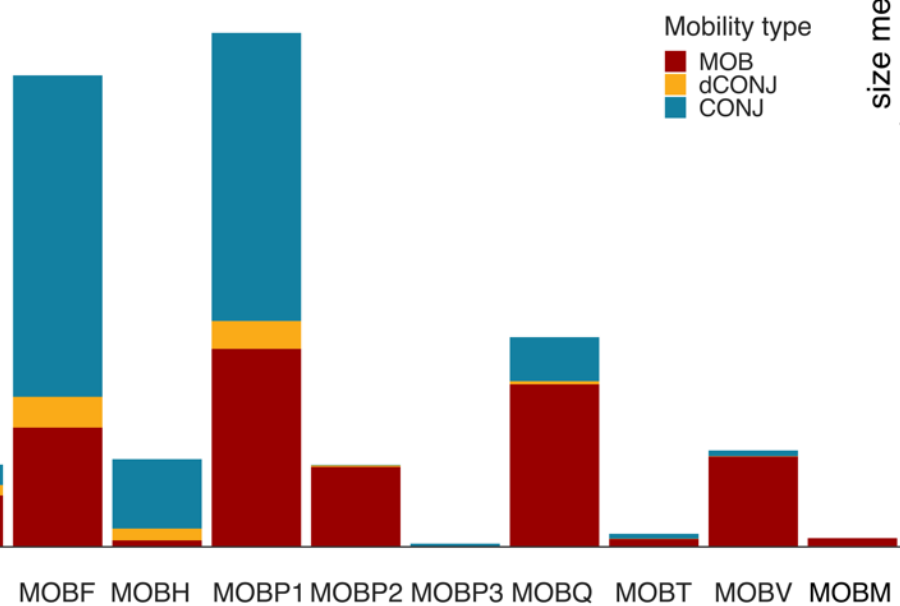

Relaxase family

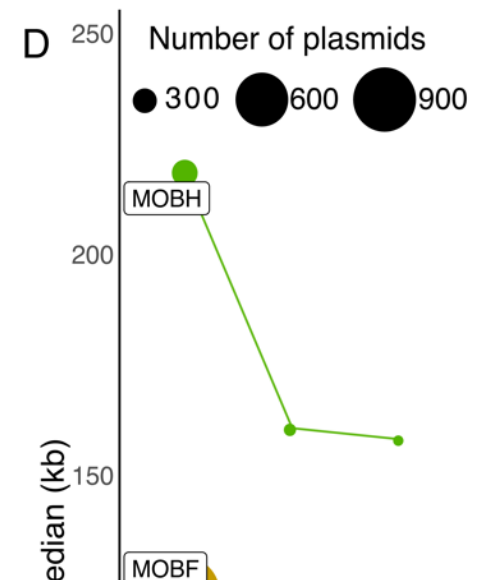

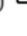

CONJ dCONJ MOB

Mobility type

Figure 1: Plasmid abundance (A, C), IS density (B) and size (D) according to mobility type and relaxase class. Panel $\mathrm{C}$ shows the median plasmid size for each relaxase family and mobility type. The width of the circles indicates the frequency of the plasmids (see inset legend).

We then assessed the distribution of plasmid mobility across bacterial taxa (Figure 2). All the taxonomic groups including more than 6 plasmids had mobilizable and/or conjugative plasmids, except the Deinococcus-Thermus. Furthermore, with the exception of the Chlorobi, which has one single plasmid, all the taxa with conjugative plasmids also have mobilizable plasmids. Hence, conjugative and mobilizable plasmids are widespread in Bacteria. The absence of these plasmids in Deinococcus-Thermus (67 plasmids) might be related with the existence of a novel transfer mechanism, called transjugation (Blesa et al. 2017). Actinobacteria and Bacilli have many more mobilizable than conjugative plasmids, which could reflect either a difficulty in identifying some conjugative systems in these clades or the previously observed overrepresentation of ICEs over conjugative plasmids in these clades (Guglielmini et al. 2011). If the latter is correct, then mobilizable plasmids in these clades might often be mobilized by ICEs. Alternatively, it has been suggested that non-conjugative plasmids in Staphylococcus spp. could be mobilized in trans by conjugative plasmids (Ramsay et al. 2016) or by transduction 
(Humphrey et al. 2021). Finally, given the frequency of mobilizable and conjugative plasmids, we searched to understand how often they co-occur. We found that $46.4 \%$ of the pMOBs are in genomes that encode at least one conjugative system. Hence, many pMOBs are in cells with a conjugative element and the others may eventually meet one by conjugation because most

297 clades encode both types of plasmids.

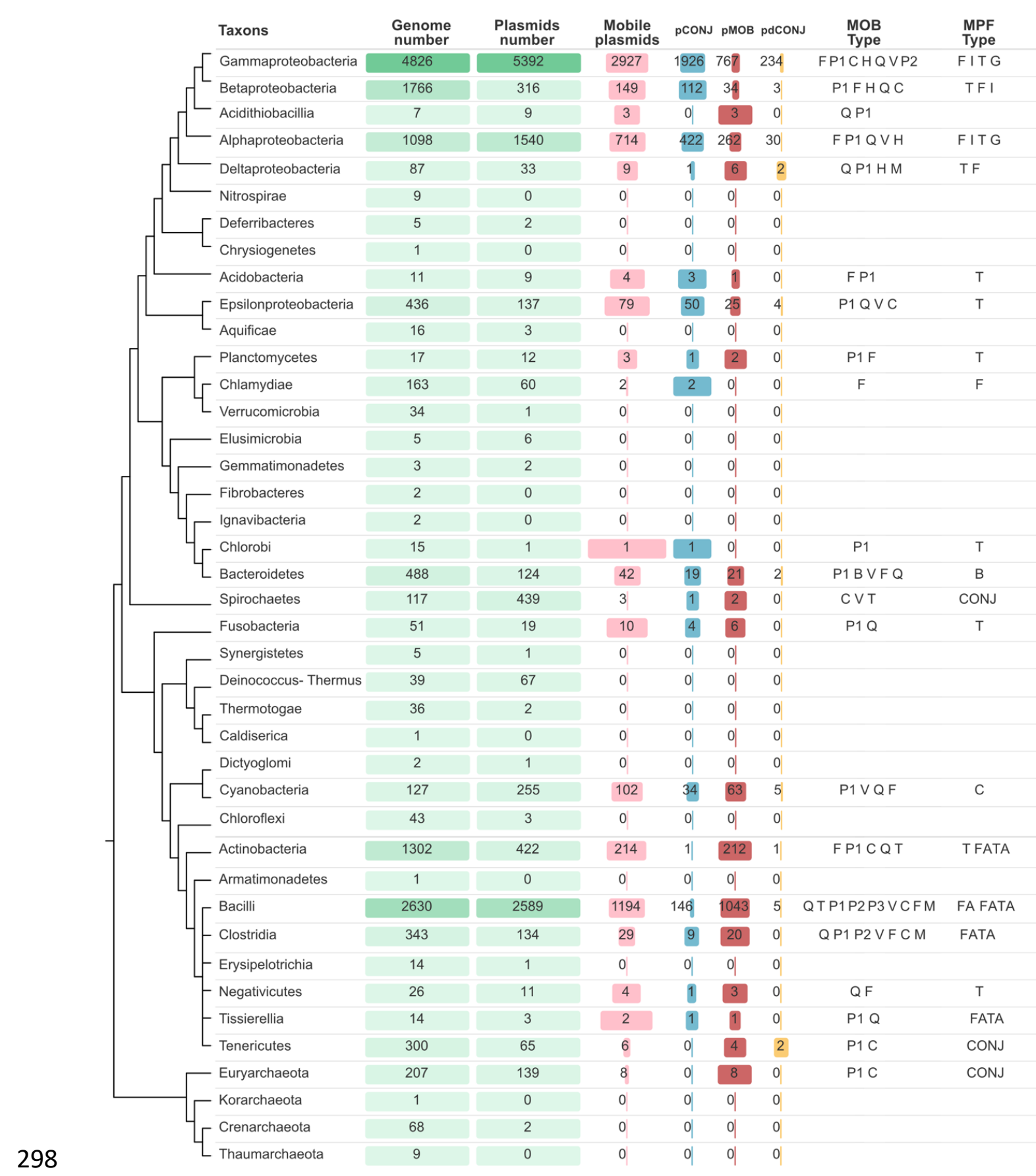

299 Figure 2: Overview of plasmids and their mobility across the tree of life. The plasmids were grouped according to their NCBI taxonomic classification of the host bacteria. This sketch tree was drawn from the compilation of

301 different published phylogenetic analyses (Denise et al. 2019). 
The promiscuity of relaxases may favor transfer of pMOB

303 While relaxases from conjugative systems are expected to have co-evolved tightly with their

304

305

306

307

308

309

310

311

312

313

314

315

316

317

318

319

320

321

322

323

324

325

326

327

328

329

330

331

332

333

334

335

336

337

338

cognate T4SS, those of pMOBs may have evolved the ability to interact with different systems to maximize their chances of transfer. To study this hypothesis, we analyzed the distribution of relaxases. Most relaxase classes are present across bacterial phyla (Figure 2). For example, the $\mathrm{MOB}_{\mathrm{p}}$ class was found in Firmicutes, Proteobacteria and Bacteroidetes. In contrast, MPF types tend to be associated with specific taxa, in agreement with (Guglielmini et al. 2013), which suggests that plasmids with the same relaxase class might be transferred by different MPF types. To study the compatibility between relaxases and T4SS we studied their co-occurrence in plasmids, since it seems reasonable to assume that in most cases the relaxases and the T4SSs carried by the same plasmid can interact. When a plasmid encodes more than one relaxase, all possible MPF TYPE$_{\text {MOB }}$ M $_{\text {CASS }}$ combinations were taken into consideration. This analysis shows that the most frequent classes of relaxases are associated with the largest number of MPF types, but these associations are not random. For example, MOBP1 is found in plasmids with 6 different MPF types, but is preferentially associated (61.9\% of the total) with MPFT or MPF. Similarly, $\mathrm{MOB}_{\mathrm{F}}$ is mostly associated with $\mathrm{MPF}_{\mathrm{F}}$ and $\mathrm{MPF}_{\mathrm{T}}$ (Figure S2). These results suggest that relaxases can work in combination with a diversity of MPF types, but exhibit preferential associations with some of them.

If plasmids encode multiple relaxases to increase the range of T4SS with which they can interact, one might expect these relaxases to be from different MOB classes to increase the diversity of interactions with MPFs. Among the 11386 plasmids, only 258 encode two relaxases and 33 encode more than two. The frequencies of these co-occurrences are similar among pCONJs and pMOBs. The most frequent relaxases of pMOBs (those retrieved by MOBV/MOBP2/MOBQ HMM profiles) co-occur more frequently in pMOBs than in conjugative plasmids. In contrast, relaxase classes found in both pCONJs and pMOBs, such as $\mathrm{MOB}_{\mathrm{F}}$ and $M O B_{P 1}$, or those that are mostly found in conjugative plasmids $\left(\mathrm{MOB}_{\mathrm{H}}\right)$ co-occur more in conjugative plasmids. This suggests that the co-occurrence of relaxases in conjugative plasmids is not often caused by fusions of pMOBs and pCONJs. Otherwise, these plasmids would have one of relaxase of each type. The co-occurrences of relaxases were as frequent within (153) as between (123) different $M O B$ classes (Figure $3 A, \chi^{2}=3.2609, d f=1, P=0.071$ ). The $M O B \vee$ class is an extreme case of self-co-occurrence; it co-occurs in 24 replicons while it occurs with other relaxases in only 11 replicons. Since these results were not suggestive of maximization of the diversity of relaxases co-occurring in plasmids, we tested if they reflected the deep evolutionary relations between relaxase families using profile-profile alignments (Figure 3B). The relaxase classes $\mathrm{MOB}_{\mathrm{F}}, \mathrm{MOB}_{\mathrm{H}}, \mathrm{MOB}_{\mathrm{T}}, \mathrm{MOB}_{\mathrm{M}}$ and $\mathrm{MOB}_{\mathrm{C}}$ showed no significant sequence homology with other classes, whereas the others were related with $\mathrm{MOB}_{\mathrm{P} 1}$. While fitting previous studies (Garcillan-Barcia et al. 2009), this does not show clear parallels between sequence similarity 
and co-occurrence in the same plasmid. Overall, these results suggest that plasmids with multiple relaxases do not seem to result systematically from the co-integration of pMOB and pCONJ neither of the optimization of the diversity of possible relaxases.
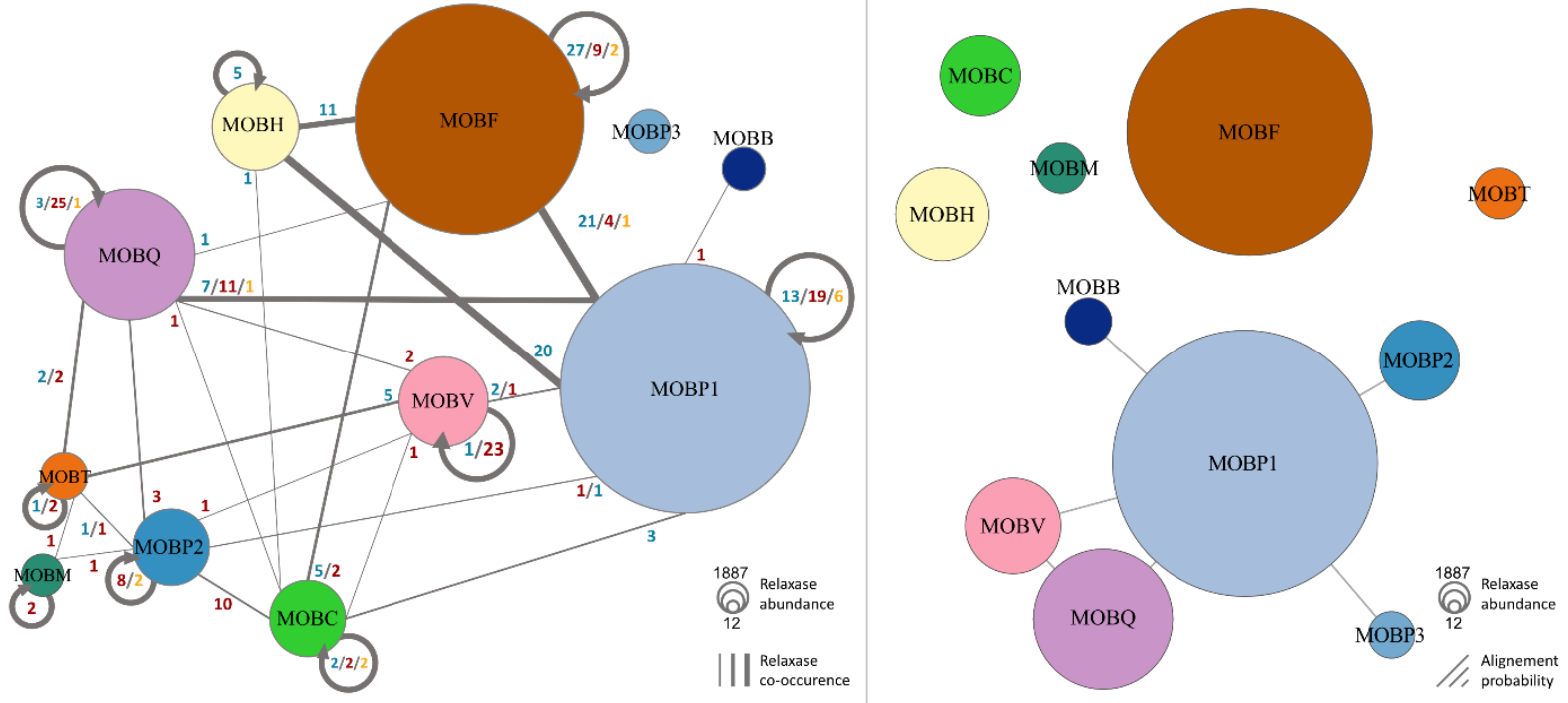

Figure 3: Associations between relaxases. Panel A. Co-occurrence (edges) of relaxases within the same plasmid. The numbers in blue, red and orange represent the number of co-occurrences found in pCONJ, pMOB and pdCONJ, respectively. Panel B. Representation of the results of profile-profile alignments between classes of relaxases. Edges represent bi-directional alignments between HMM profiles, and their length represents the probability of the alignment (short links represent a higher probability (up to 96\%) and long links represent lower probability (down to 76\%)). Are only represented alignments having a probability superior to $70 \%$ and an e-value inferior to $10^{-4}$.

\section{Plasmid size and gene repertoire evolve fast relative to the relaxase phylogeny}

To start the study of the evolution of plasmid mobility we focused on the relaxases retrieved by

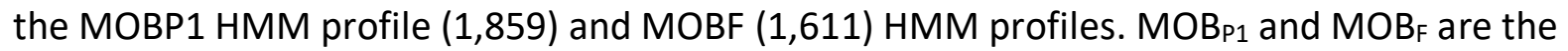
most abundant relaxase classes, they rarely co-occur in the same plasmid, they are sufficiently conserved to be good phylogenetic markers, and they are frequent in mobilizable and conjugative plasmids. Some of the relaxases were nearly identical. To remove redundancy and accelerate computational analyses while keeping the genetic diversity of the dataset, we clustered the proteins that aligned over at least $99 \%$ of the length with at least $99 \%$ identity and picked one representative per group. This resulted in $850 \mathrm{MOB}_{\mathrm{P} 1}$ and $735 \mathrm{MOB}_{\mathrm{F}}$ relaxases that were used to make phylogenetic reconstructions by maximum likelihood (see details in Methods). These proteins are representative of their clusters because most of them include only plasmids from one single mobility type ( $94.9 \%$ of $\mathrm{MOB}_{\mathrm{P} 1}$ and $92.5 \%$ of $\mathrm{MOB}_{\mathrm{F}}$ ). The two trees are well supported in most branches. In both cases, there is a clear tendency for closely related relaxases to be in plasmids with similar MPF types. This leads to large clades overrepresenting one MPF type (Figure 4). In the $\mathrm{MOB}_{\mathrm{P}}$ tree there is an overrepresentation of $\mathrm{MPF}_{\mathrm{T}}$ that seems to suggest this was the original MPF associated with this relaxase. The $\mathrm{MOB}_{\mathrm{F}}$ tree is 
366 too variable at the basal clades to make any kind of conclusion regarding the ancestral

367 associations. Differences in plasmid size are very pronounced across the tree, since many

368 plasmids that are close in the tree exhibit wide variations in size. Hence, plasmid size varies fast

369 relatively to the protein sequence of the relaxases.

370 Both phylogenetic trees divide the plasmids into large clades dominated either by pMOBs or

371 pCONJs, but rarely containing similar numbers of both. Sister-groups are very distinctive and

372 often separate clades of many large conjugative plasmids from those including many small

373 mobilizable elements (although there is one group of large pMOBs in each tree). The clades

374 with a majority of conjugative elements have many pMOBs and pdCONJ, but clades with a

375 majority of mobilizable elements have very few pCONJ. pdCONJ are scattered across the tree,

376 typically very close to conjugative plasmids. This is consistent with the hypothesis that such

377 elements derived recently from conjugative plasmids by gene loss. Overall, these trees suggest

378 early separations between sub-families of relaxases that tend to be specific for mobilizable or

379 for conjugative elements. They also suggest more frequent conversion of conjugative elements

380 into other type of plasmids than the other way around. 


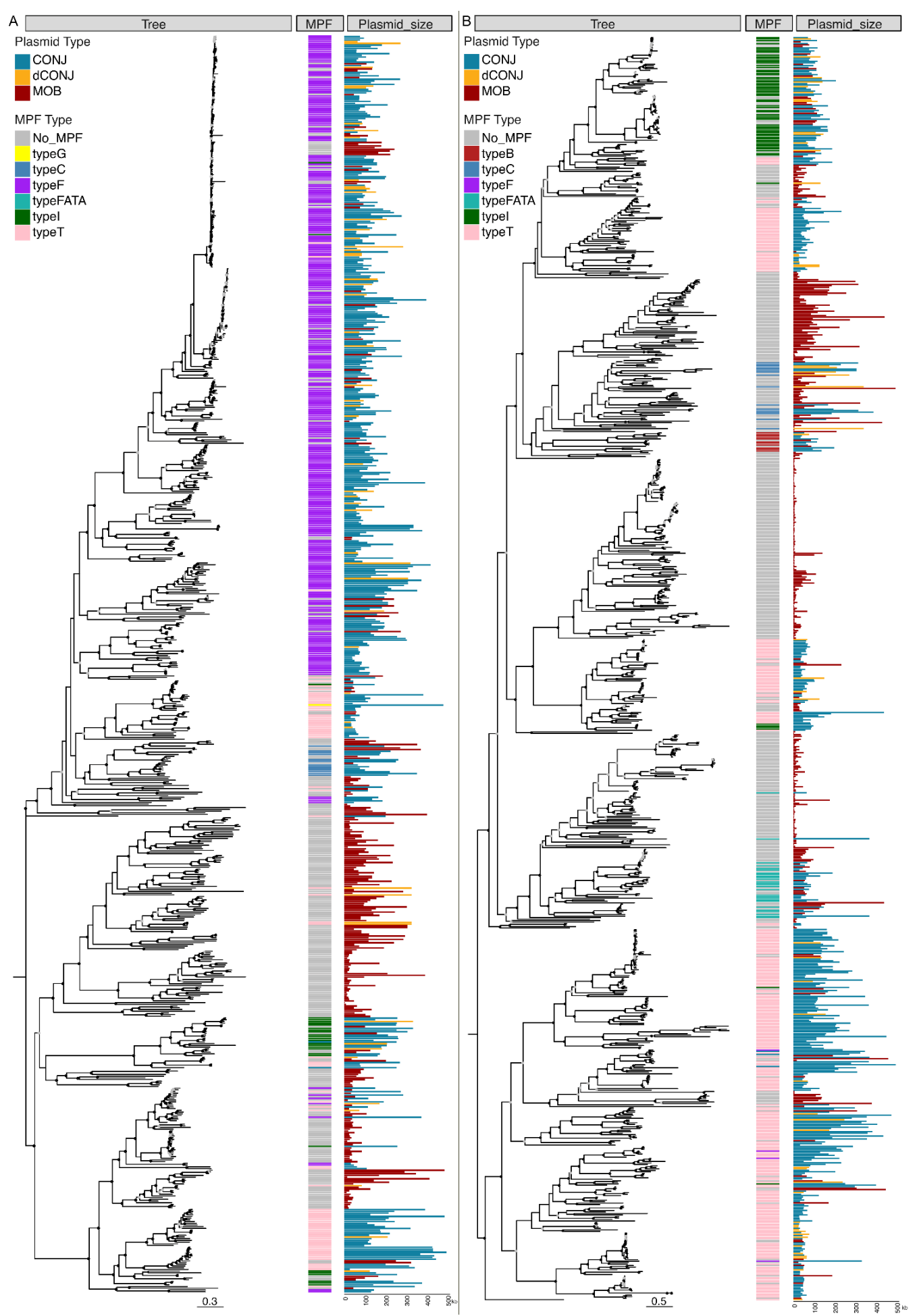

Figure 4: Phylogenetic trees of $\mathrm{MOB}_{\mathrm{F}}$ relaxases and $\mathrm{MOB}_{\mathrm{P} 1}$ relaxases. Ultrafast bootstrap values superior to 75 are shown with a light grey circle and values superior to 90 with a black circle. The trees were rooted using the midpoint root. Panel A: The phylogenetic tree was built using $735 \mathrm{MOB}_{\mathrm{F}}$ proteins with maximum likelihood with IQTree (model WAG+F+R10 and 1000 ultrafast bootstraps (Nguyen et al. 2015)). Panel B: The phylogenetic tree was built using $850 \mathrm{MOB}_{\mathrm{p}}$ proteins (all retrieved with the MOBP1 HMM profile) with maximum likelihood with IQTree (model VT+F+R10 and 1000 ultrafast bootstraps). 
We then assessed the variations in gene repertoires for the 5,512 plasmids with the two relaxases. We calculated the weighted gene repertoire relatedness (wGRR) between each pair of plasmids, which is the number of reciprocal best hits between two plasmids weighted by their sequence similarity and divided by the number of genes of the smaller element (see Methods). A wGRR close to 1 means that plasmids are very similar (or one is a subset of the other) and a wGRR of 0 indicates no homology. Around 2500 comparisons between plasmids show very high wGRR, but most pairs of plasmids have low wGRR (Figure S3). We searched for pairs of plasmids with high wGRR but different families of relaxases. Such cases were extremely rare. We found 22 cases, of which none implicated a pair of $\mathrm{MOB}_{\mathrm{P} 1}-\mathrm{MOB}_{\mathrm{F}}$ plasmids. Hence, closely related plasmids tend to have nearly identical relaxases.

Pairs of plasmids with nearly identical relaxases ( $\geq 99 \%$ identity) have similar gene repertoires (very high wGRR), except when they have different types of mobility (Figure 5, Figure S4). To understand how PTUs vary at these short evolutionary scales, we assessed their variability in clusters of plasmids having $>99 \%$ identical relaxases from different MOB classes. In most cases the PTU is the same across the cluster. Yet, we found different PTUs in $18.2 \%$ of the 307 clusters analyzed, $17.5 \%$ of the $\mathrm{MOB}_{\mathrm{P} 1}$ and $15.6 \%$ of the $\mathrm{MOB}_{\mathrm{F}}$ (Figure S5).

Diverged relaxases (<95\% identity) are associated with plasmids having wGRRs lower than $50 \%$ $\left(\mathrm{MOB}_{\mathrm{P} 1}\right)$ or $40 \%\left(\mathrm{MOB}_{\mathrm{F}}\right)$, i.e. many such plasmids are poorly related or entirely unrelated. At $50 \%$ identity between the relaxases, the average wGRR is less than $30 \%$ (even though the plasmids have necessarily the relaxase as homolog). Interestingly, the average trends in terms of WGRR are similar for pMOBs and pCONJs, suggesting comparable rates of change in gene repertoires.
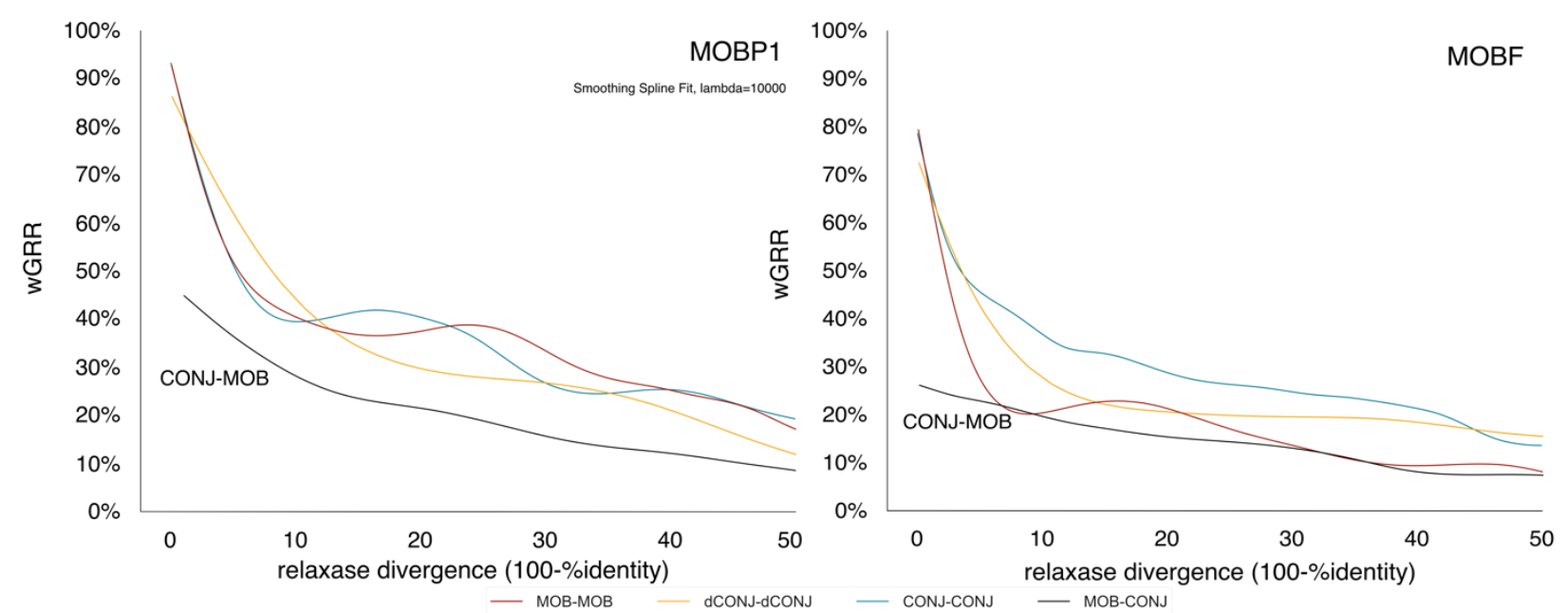

Figure 5: Relationship between relaxase protein sequence divergence and WGRR in pairs of plasmids classed in terms of mobility for $\mathrm{MOB}_{\mathrm{P} 1}$ (left) and $\mathrm{MOB}_{\mathrm{F}}$ (right). Each curve represents the smoothing spline for comparisons between: pdCONJ (yellow), conjugative (blue), mobilizable (red), and comparisons between mobilizable and conjugative plasmids (black). 


\section{Conjugative plasmids are the source of other plasmids}

417 To quantify the rates of transition between mobility types, we inferred the ancestral mobility of

418 plasmids using PastML and identified 249 terminal branches associated with a change. This

419 analysis was made using the previously presented data of $\mathrm{MOB}_{\mathrm{F}}$ and $\mathrm{MOB}_{\mathrm{P} 1}$ that we combined

420 with data from the plasmids carrying other relaxases (phylogenetic trees in Figures S12-18). We

421 focused our analysis on terminal branches because these ancestral reconstructions are more

422 accurate and pinpoint the plasmids that have recently changed in terms mobility. The most

423 common transitions are from $\mathrm{pCONJ}$ to $\operatorname{pMOB}\left(116\right.$, rate $\left.=0.166 \mathrm{Subst}^{-1}\right)$ or pdCONJ (77,

424 rate $=0.065$ Subst $^{-1}$ ) (Figure 6a). In contrast, we detected only 36 transitions from pMOB to

425

426

427

428

429

430

431

432

433

434

435

436

437

438

439

440

441

442

443

444

445

446

447

448

449

450

451

452 pCONJ and even fewer from pdCONJ to the others. We found approximately similar rates of transition from dCONJ to mobilizable (0.014), vice-versa (0.013), and pdCONJ to conjugative (0.017). These values are all much smaller than the transition rates from conjugative plasmids, confirming that conjugative plasmids are the source of most of the recent transitions between plasmid mobility types.

The comparisons between pCONJs and pMOBs with nearly identical relaxases revealed low average wGRRs (Figure 5). This suggests that transitions between mobility types, conjugative to mobilizable or vice versa, are often associated with large changes in plasmid gene repertoires. Transitions pCONJ to $\mathrm{pMOB}$ can be explained by deletions of the genes encoding the conjugative system or by translocations of a relaxase from a pCONJ to a MOBless plasmid (Figure 6D). To try to distinguish between the two types of events, we computed the distribution of WGRR for pairs of closely related pMOB-pCONJ pairs. We expected to find a bimodal distribution indicating pairs with very low wGRR originating from translocations and pairs with larger $W G R R$ originating from gene deletions. Our analysis of $M B_{F}$ and $M O B_{P 1}$ failed to uncover any obvious bi-modality (Figure S6). Without excluding the possibility that translocations occur occasionally, three arguments suggest that transition by gene deletions are frequent. First, transitions pCONJ to PMOB involve a reduction in plasmid size and in wGRR (Figure 5, Figure S7, Figure 6b), but these novel pMOBs are closer in size to pdCONJ than to the typical pMOB and much larger than the average MOBless plasmid (Figure S10, Wilcoxon test, $P<0.0001)$. Second, the novel pMOBs often still encode a few genes of the T4SS (52\% encode at least one MPF protein and $28 \%$ encode two or more), which is much more than the other pMOBs (Wilcoxon test, $\mathrm{P}<0.001$, Figure S11). Third, pCONJ to pdCONJ transitions, which are presumably very recent since the conjugation locus has not yet been fully deleted in the latter, show small differences in size and wGRR (Figure 6B). This effect is not due to differences in the length of the terminal branch where we observe transitions, since those associated with transitions pCONJ to $\mathrm{pMOB}$ and $\mathrm{pCONJ}$ to pdCONJ are not significantly different (Wilcoxon test, $P=0.130$, Figure S9). Instead, these results suggest that pdCONJ arise by partial deletions on the MPF locus without necessarily implicating immediate major changes in the plasmid gene 
453 repertoire (Figure 6D). These pdCONJ may then rapidly endure further gene deletions resulting

454 in pMOBs, a process that may be facilitated by the presence of high densities of transposable

455 elements in these plasmids (Figure 1B).

456 The results above suggest that changes in mobility are followed by further gene turnover that

457 results in low wGRR between neighbor pMOB and pCONJ in the relaxase phylogenetic tree. We

458 were particularly intrigued by the possibility that novel mobilizable plasmids could co-exist in

459 the cell with the ancestral plasmids. If the novel pMOB maintain the replication system they

460 had when they were conjugative, they should become incompatible with the closely related

461 pCONJ and thus not be stably kept in the same cells. But this would affect the viability of the

462 novel pMOB, since the closest related pCONJ are those that are most compatible with its

463 relaxase. We therefore typed the replicons and analyzed if those that recently changed in terms

464 of mobility also changed in terms of replication initiation proteins (Inc type). This is often the

465 case among the few such plasmids that could be typed: $68 \%$ have an Inc type different from the

466 one of the closest related pCONJ (17/25). The latter also have a much smaller wGRR than those

467 that maintained the same Inc type (Figure 6C). Such changes may be part of the broader

468 changes resulting in rapid decrease of WGRR values after transition from pCONJ to pMOB. 

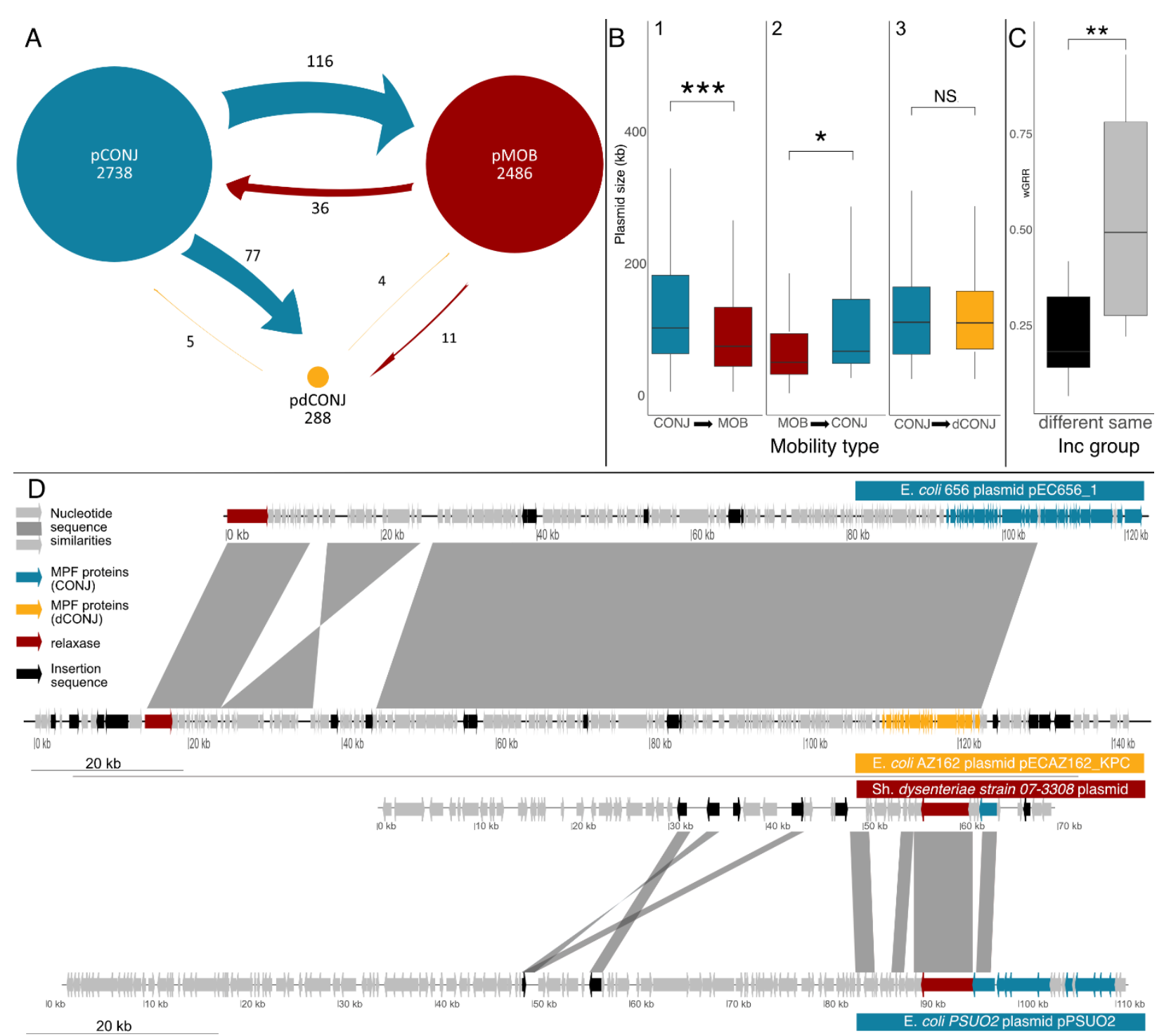

Figure 6: Characterization of mobility transitions. A. Transitions inferred in terminal branches of the relaxase phylogenetic trees. Arrows and numbers represent the direction and number of transitions, respectively. The size of circle indicates the abundance of each plasmids type. B. Boxplots representing the size of plasmids that recently changed in terms of mobility (in comparison to the sister-taxa plasmid in the relaxase tree having the ancestral state). Boxplot 1,2 and 3 represent the size distribution associated with transitions from pCONJ to pMOB, from pMOB to $p C O N J$ and from pCONJ to pdCONJ respectively. C. Boxplot of the wGRR between pairs of plasmids where one recently changed from $\mathrm{pCONJ}$ to $\mathrm{pMOB}$. The grey (black) boxplot represents the wGRR for plasmids of the same (different) replicon type. D. Comparison between selected plasmids, plotted using GenoPlotR v.0.8.10 (Guy et al. 2010), based on Blastn analysis of the plasmid nucleotide sequences (evalue $<10^{-4}$ and alignment length $>1000$ bp). Top. Comparison between a pdCONJ (yellow) and a conjugative plasmid (blue). Bottom. Comparison between a mobilizable (red) and a conjugative (blue) plasmids. For clarity, we only represent regions of homology larger than $1 \mathrm{~kb}$ with more than $50 \%$ identity. Genes involved in the plasmid mobility (T4SS and relaxase(s)) are highlighted. Tests of differences (Wilcoxon paired-tests): ${ }^{* *}(\mathrm{P}<0.001),{ }^{* *}(\mathrm{P}<0.01),{ }^{*}(\mathrm{P}<0.05)$, NS (nonsignificant). 
487 The previous results suggest that conjugative plasmids are often the source of other plasmids.

488 To quantify the persistence of the different types of plasmids we analyzed the phylogenetic 489 trees of relaxases. Instead of focusing only on terminal branches of the phylogenetic trees 490 alone, we computed the shortest patristic distances, i.e., the distances between taxa in the 491 tree, between all plasmids of different mobility types. This allows to study plasmid lineages that 492 are very persistent (rarely changing). We then computed the cumulative distribution functions 493 of these patristic distances for each type of mobility (CDF) (Figure 7). A rapid initial increase in 494 the CDF with increasing patristic distance indicates that the state is not very persistent, i.e. there are plasmids with different types of mobility at small patristic distances. In contrast, a 496 slow increase in the CDF means that the closest plasmid with a different mobility type is usually 497 distant in the tree. The results for $\mathrm{MOB}_{\mathrm{P} 1}$ and $\mathrm{MOB}_{\mathrm{F}}$ are qualitatively similar and show that dCONJ plasmids are always close to other types of plasmids in the tree of relaxases. This is consistent with the frequent transitions from pCONJ and shows that these pdCONJ are transient, either because they rapidly change again their type or because they are lost from populations. Conjugative plasmids have intermediate CDFs, which is consistent with the observation that they are frequently the source of pdCONJ and pMOBs. In contrast, pMOBs exhibit a steep increase in CDF with initial increase in patristic distances, which are then followed by a much slower increase of the CDF for larger patristic distances. This is consistent with the observation that a fraction of $\mathrm{pMOBs}$ derived recently from pCONJ. The remaining pMOBs are placed in clades of the relaxase phylogenetic tree where there are almost only pMOBs, explaining the higher persistence of this mobility type relative to the others. the abovementioned patterns of change differ among relaxases associated with either one or the other, but not both, types of plasmid mobility, we analyzed the CDF for MOBv (mostly

511 mobilizable plasmids) and $\mathrm{MOB}_{H}$ (mostly conjugative plasmids). The results for $\mathrm{MOB}_{\mathrm{V}}$ mirror

512 those of $\mathrm{MOB}_{\mathrm{P} 1}$ and $\mathrm{MOB}_{\mathrm{F}}$, with the exception that the CDF increases faster for conjugative

513 plasmids and slower for mobilizable. This suggests that plasmids with relaxases specialized in

514 mobilizable plasmids rarely become conjugative. In contrast, the CDF increases much slower for conjugative elements with $\mathrm{MOB}_{\mathrm{H}}$, suggesting that they rarely lead to mobilizable plasmids.

516 These results are consistent with a specialization of certain relaxases in plasmids with specific 517 types of mobility. 

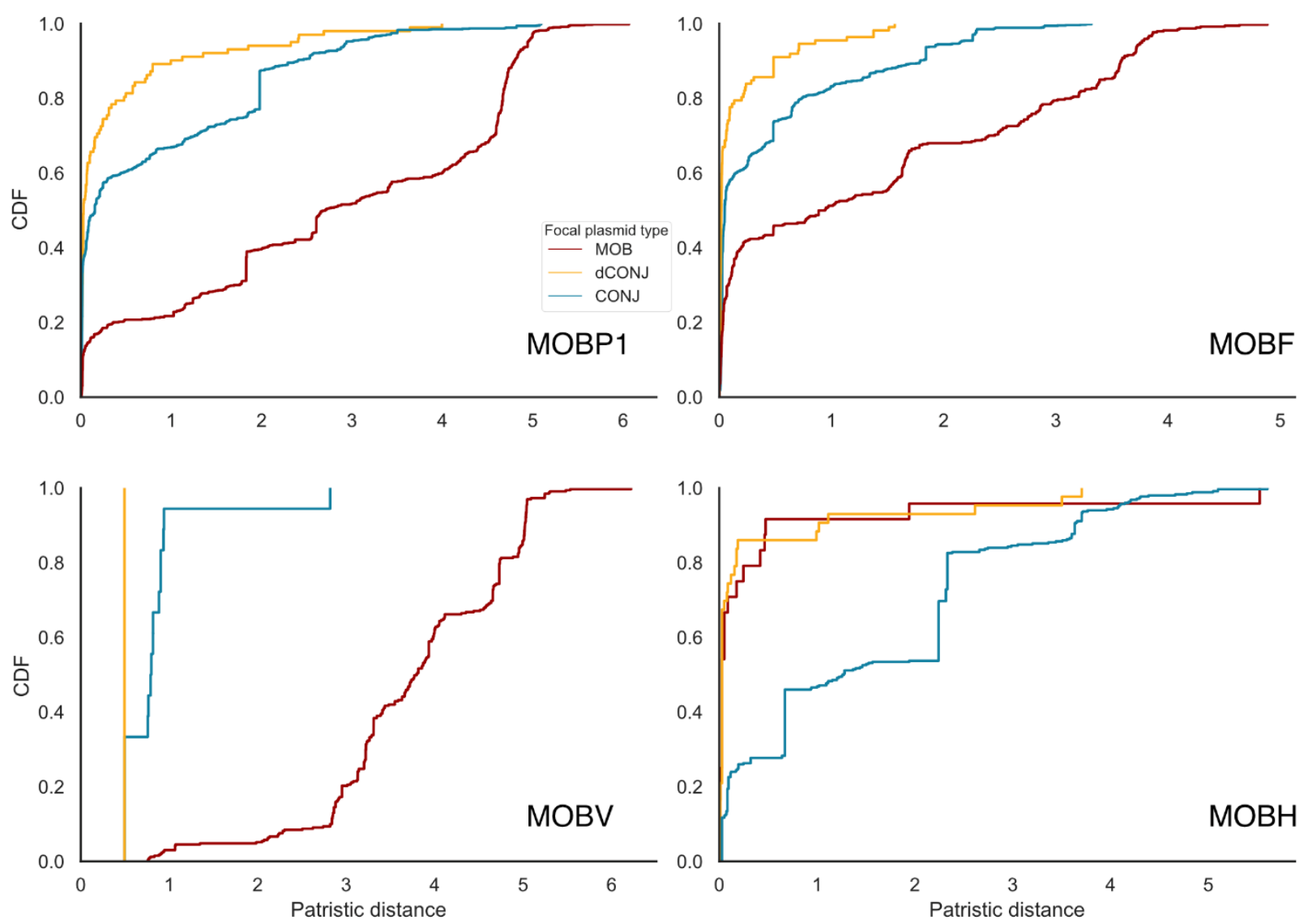

Figure 7: Cumulative distribution function (CDF) of the minimal patristic distances in the relaxase phylogenetic trees from a relaxase to another relaxase of a different mobility type. When the CDF approaches 1 for low patristic distances, this means that all relaxases are close to a relaxase of a plasmid with a different mobility type in the tree.

\section{Transitions to and from MOBless plasmids are frequent}

524 In the precedent sections we focused on conjugative and mobilizable plasmids because they can be analyzed in terms of the presence and evolution of the relaxase. Yet, around half of the plasmids lack a recognizable relaxase (MOBless, Figure $1 \mathrm{~A}$ ). If plasmids can change between conjugative and mobilizable types, they may certainly also gain or lose the relaxase. The study of the transitions to and from MOBless plasmids is difficult because they lack conserved phylogenetic markers. The only alternative to the relaxase, the replication initiation genes, are very diverse, often unrecognizable, and they can be protein or RNA genes, making deep evolutionary studies in a phylogenetic framework impossible. To study the transition between MOBless and other plasmids, we identified non-redundant pairs of MOBless-(pCONJ, pMOB, pdCONJ) plasmids that presumably diversged recently because their wGRR is high (superior to 0.75 , see details in Methods). We detected 345 mobile plasmids that had one closely related MOBless plasmid: 154 pCONJs, 172 pMOBs and 19 pdCONJs (Figure 8). One might have 
be small, and it only takes gain/loss of a relaxase to transition from one into the other. Yet, this is not the case and there is no significative difference between the number of pCONJ/pMOBless pairs (44.5\%) and pMOB/pMOBless pairs $(50 \%)\left(\chi^{2}=2.8209, \mathrm{df}=1, \mathrm{P}=0.09\right)$. Hence, transitions from or to MOBless plasmids may be quite common for all plasmid mobility types.

To assess the consequences of these transitions, we compared the sizes of the plasmids of each pair. When the analysis was stratified by plasmid mobility type, it revealed that MOBless plasmids are significantly smaller than the other element of the pair independently of the mobility type of the latter (Figure 8). This suggests that transitions are associated with gene losses in the novel MOBless plasmids or gene acquisition in the others (when transition occurs in the opposite sense). Alternatively, these patterns could result from co-integration of a MOBless plasmid in a plasmid with a relaxase. Yet, in this case one would expect very large differences in sizes between the pMOBless and the cointegrate. Instead, the size of pMOBless is often only slightly smaller than that of the other plasmid. This is more consistent with a model of gene deletion resulting in a transition to pMOBless.
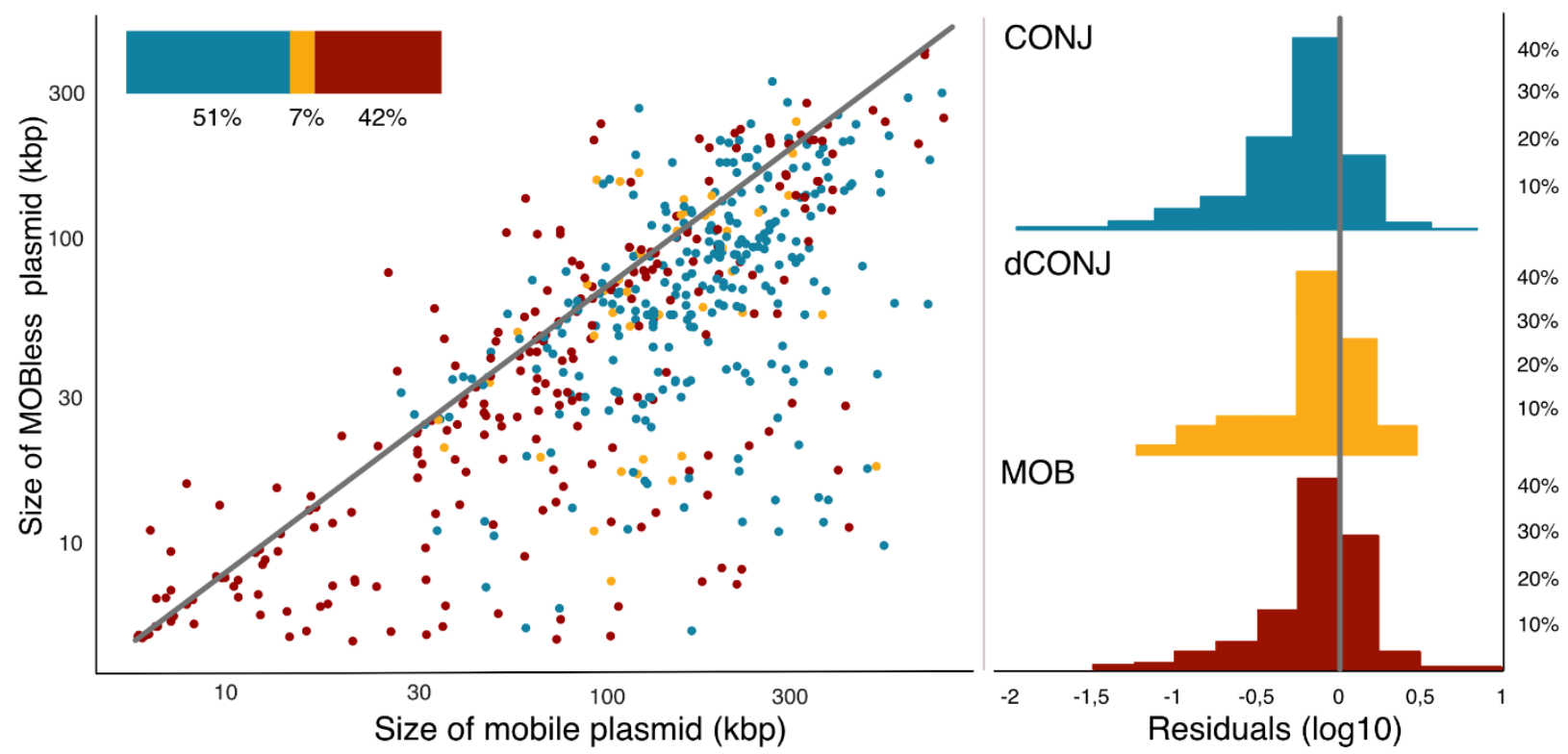

Figure 8: Relationship between the size of mobilizable or conjugative plasmid and the closest related MOBless plasmid. Each data point in the scatter plot represents the size of pairs of plasmids that are very similar (wGRR>0.75) and one is pMOBless whereas the other isn't. Blue, red and yellow dots represent pairs between MOBless and CONJ, MOB and dCONJ plasmids respectively. The histograms on the right represent the distribution of the $\log 10$ of the residuals (distance to the identity line in grey). All three distributions have an average significantly lower than zero (all Wilcoxon tests, $\mathrm{P}<0.0001$ ).

\section{Conservation of PTUs when mobility changes}

To understand the interplay between changes in mobility and changes in PTUs, we focused on the $\mathrm{MOB}_{\mathrm{F}}$ subtree of the $\mathrm{MOB}_{\mathrm{F} 12}$ family of plasmids from $E$. coli and Shigella spp. because they are numerous and well characterized (Fernandez-Lopez et al. 2016). We analyzed these 
562 plasmids using pairwise average nucleotide identity (ANI) comparisons. This resulted in a graph

563 where we could plot the mobility of the plasmids and the PTUs (Figure 9A). In the network one

564 can distinguish those plasmids that have a different mobility type tend to be at the outer edge

565 of the main PTU (PTU- $\left.\mathrm{F}_{\mathrm{E}}\right)$ or are directly classified as different PTUs. Depending on the effect of

566 changes in mobility on gene repertoires, they may result in conservation of the plasmid PTU, or

567 its budding out of the original PTU, sometimes resulting in new PTUs (such as E5, Fsh, E41, etc.).

568 In this specific case, the emerging PTUs seem to be associated with specific hosts (e.g. PTU-F

569 to Shigella spp., and PTU-E5 to E. coli O157:H7), which may be an indication that new PTUs

570 emerge by change of mobility and adaptation to new hosts. It should also be mentioned that

571 relaxase genes within the members of PTU-F may have down to $90 \%$ sequence identity

572 (example, R1 vs R100), while transitions to the new PTUs occur while their relaxases remain

573 more than 95\% identical to some members of PTU-FE (examples, to PTU-E78 or PTU-E32).

574 The largest component of the ANI graph corresponds to the PTU-FE plasmids, which a PTU

575 dominated by conjugative plasmids. We used ACCNET to analyse the ORFeome shared by the

576 members of this PTU (Figure 9B). Results show that plasmids that changed in terms of mobility

577 are scattered in the network. Hence, there are also many transitions of mobility type at this

578 micro-evolutionary scale, although the changes in gene repertoires are not sufficiently large as

579 to expel the plasmid from the PTU. Interestingly, these recent plasmid variants show sizes that

580 are much larger than those of most mobilizable of MOBless plasmids. In this PTU, the average

581 size of the presumably ancestral pCONJ plasmid is $117.4 \mathrm{~kb}$, whereas those of the novel types is

582 very close: $118 \mathrm{~kb}$ for pdCONJ, $112 \mathrm{~kb}$ for pMOB and $95.3 \mathrm{~kb}$ for pMOBless. This analysis at the

583 micro-evolutionary scale is thus consistent with gene deletions being the cause of many

584 transitions from pCONJ.

585 We showed above that when $\mathrm{MOB}_{\mathrm{F} 12}$ plasmids change in terms of mobility type they endure

586 significant changes in gene repertoires. This means that their PTUs may also change. Indeed, we

587 observed that PTUs of pairs of pMOBless-(pCONJ,pdCONJ,pMOB) are often different (Figure

588 S19). When these pairs have high wGRR values (>95\%), $4 \%$ of the pairs exhibit transitions

589 between known PTUs (as shown above for some examples on PTU-FE). When wGRRs are lower

$590(75-80 \%)$ these transitions increase to $9.4 \%$. Hence, transitions in terms of mobility impact the

591 taxonomical classification of plasmids when they implicate large changes in gene repertoires. 

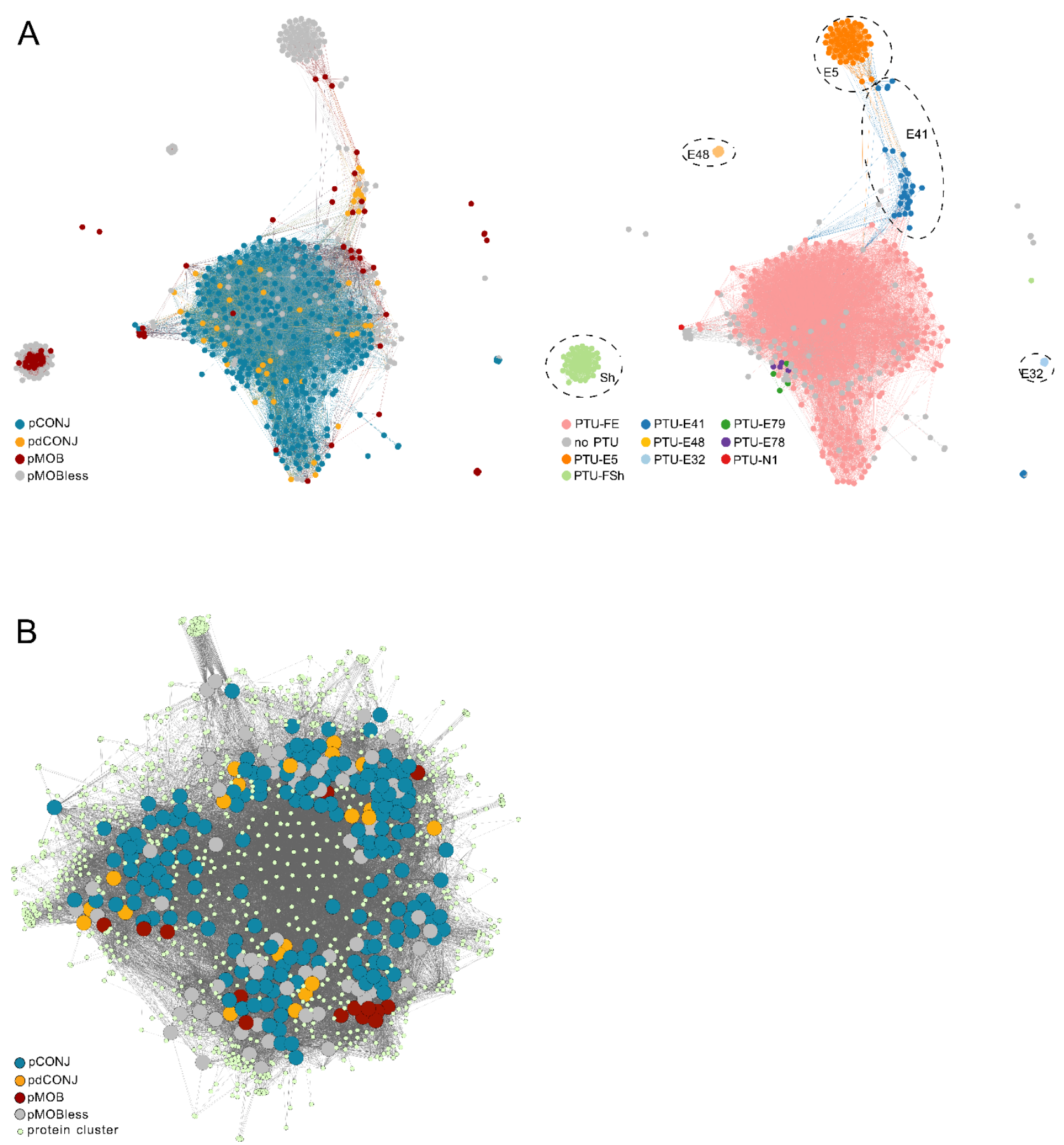

Figure 9: A. ANI similarity network of the $\mathrm{MOB}_{\mathrm{F} 12}$ plasmid family from $E$. coli and Shigella spp. The genomic relatedness of 353 transmissible $\mathrm{MOB}_{\mathrm{F} 12}$ plasmids hosted in E. coli and Shigella spp., and 140 MOBless plasmids belonging to the same PTUs was estimated by pairwise ANI calculations. The nodes correspond to plasmids and edges indicate non-null ANI scores. At the left, nodes are colored by their mobility type, and at the right, by their PTU. "no PTU" means non-assigned PTU. In the right panel, PTUs that are separated from the main group (PTU- $\mathrm{F}_{\mathrm{E}}$ ) are surrounded by a circle. B. ORFeome network of the PTU-FE plasmids. The proteins of 270 PTU-FE plasmids were clustered at $95 \%$ identity and $80 \%$ coverage. Whenever a plasmid has a member in a protein cluster, an edge is linking them. Homologous protein clusters present in a single plasmid were removed from the figure. Plasmids

601 are colored by their mobility type. 


\section{Discussion}

603 In this study we aimed at understanding the evolution of plasmid mobility. We focused on 604 plasmids encoding relaxases because these are expected to be able to transfer horizontally.

605 Furthermore, the relaxase is a well-conserved protein that allows the study of plasmid

606 evolution using a rigorous phylogenetic framework, including rates and directions of change in 607 the type of mobility. Conjugative systems, and thus relaxases, appeared early in the history of 608 life (Guglielmini et al. 2013). Since plasmids with a relaxase can only be mobilizable if there is a 609 conjugative system available, one may presume that conjugative plasmids arrived first and mobilizable plasmids arrived later. Since a gene deletion is sufficient for a conjugative plasmid to become mobilizable, pMOBs may have arisen very quickly after pCONJ. This fits the

612 observation of large ancient clades of relaxases specific to mobilizable plasmids in the $\mathrm{MOB}_{\mathrm{P}}$

613 and $\mathrm{MOB}_{\mathrm{F}}$ trees. It is also consistent with the existence of $\mathrm{MOB}_{\mathrm{P}}$ (specifically relaxases retrieved 614 with the MOBP2 HMM profile) and MOB families that are distantly related with hits of the 615 MOBP1 HMM profile and are almost exclusively found in mobilizable plasmids. Importantly, 616 none of the relaxase classes unrelated with $\mathrm{MOB}_{\mathrm{P}}\left(\mathrm{MOB}_{\mathrm{H}}, \mathrm{MOB}_{\mathrm{F}}, \mathrm{MOB}_{\mathrm{M}}, \mathrm{MOB}_{\mathrm{C}}\right)$ is specific to 617 mobilizable plasmids. The apparent exception is $\mathrm{MOB}_{T}$ that is extremely rare in conjugative 618 plasmids, but very frequent in ICEs (Soler et al. 2019). These results suggest that a few initial 619 classes of relaxases were present in conjugative plasmids and by diversification and specialization some families became specialized in mobilizable plasmids. The existence of specialized relaxase families, manifested as specific clades in the $\mathrm{MOB}_{\mathrm{F}}$ and $\mathrm{MOB}_{\mathrm{P}}$ trees,

622 suggests adaptation of these proteins to mobilizable plasmids. The alternative hypothesis, that

623 they arose in a type of plasmid and were never transferred into another type of plasmid seems extremely unlikely given the observed rate of change of their gene repertoires, the observed transitions between types in some clades of the trees, and the previously known gene flow between plasmids (Eberhard 1990; Revilla et al. 2008; Redondo-Salvo et al. 2020).

Why would there be a specialization of relaxases? One expects tight co-evolution between the T4SS and the cognate relaxase of conjugative plasmids because they are self-mobilizable and the two components have co-evolved to interact efficiently. For example, the pCONJ RK2 and RP4 have very similar origins of transfer, but are unable to mobilize each other (Fürste et al. 1989). In contrast, mobilizable plasmids require a conjugative element to transfer and may have evolved to interact with very different conjugative systems. Accordingly, $\mathrm{MOB}_{\mathrm{Q}}$ family plasmids can be transferred by at least the MPF and MPFT systems (Garcillan-Barcia et al. 2019), RSF1010 is mobilized by MPF, MPF and other uncharacterized systems (Meyer 2009), MOBP5 mobilizable plasmids (ColE1) are mobilized by MPFF and MPF, plasmids (Cabezón et al. 1997),

636 MOBV1 $_{V_{1}}$ mobilizable plasmids, such as pMV158, are mobilized by MPFT and MPFFATA (Lorenzo-

637 Díaz et al. 2014), and SGI1 genomic islands from Salmonella enterica can be mobilized by IncA 638 and IncC plasmids (Szabó et al. 2021). A recent study showed that IncQ plasmids, which are 
639 pMOB, have exceptionally large host range (Stalder et al. 2019). Finally, while the pCONJ R388

640 and RP4 cannot mobilize each other, both can mobilize the unrelated pMOBs RSF1010 and

641 ColE1 (Cabezón et al. 1994). The flexibility of pMOB relaxases may result in less efficient

642 interactions between specific T4SS (Sastre et al. 1998). Hence, relaxases of mobilizable plasmids

643 would evolve to interact with many different T4SS at the cost of interacting less efficiently with

644 any single one.

645 Our analysis showed that gene repertoires change very fast, relative to the evolution of

646 relaxases. The pace of change is further accelerated when there is a change in mobility type,

647 raising the question of the genetic mechanisms driving these transitions. Transitions between

648 types of mobility can be due to accretion/deletion of genetic material or translocation of a

649 relaxase to another replicon. It should be noted that given the rapid pace of gene repertoire

650 evolution, it is intrinsically difficult to distinguish large translocations from plasmid co-

651 integrations. Still, several arguments suggest that most transitions of pCONJ to other types of

652 plasmids tend to result from deletion of genetic material. First, the relaxase gene is not mobile

653 in itself and the homology between closely related plasmids of different type usually extends

654 beyond the relaxase. Second, the WGRR is rarely close to zero as expected if the putative

655 translocation affected only the relaxase. Third, many conjugative systems become pdCONJ, and

656 this seems clearly the result of small deletions. It is possible that many transitions between

657 conjugative and $\mathrm{pMOB}$ have rapidly passed by a pdCONJ intermediate state that we were no

658 longer able to observe in many cases. Accordingly, pdCONJ and novel pMOB are much larger

659 than the other $\mathrm{pMOB}$ and closer to the size of conjugative plasmids.

660 The high rates of transition from conjugative to mobilizable plasmids only requires gene loss,

661 which is frequent in bacteria (Mira et al. 2001). Deletions are stimulated by transposable

662 elements (Cerveau et al. 2011), which we found to be more abundant in pCONJ and especially

663 pdCONJ elements. Hence, conjugative plasmids are more likely sources of other plasmids than

664 the converse. Selection may impact the success of gene deletions (Koskiniemi et al. 2012). Both

665 phylogenetic trees and the analyses of patristic distances between all pairs of plasmids reveal

666 that many transitions from pCONJ are very recent (Figures 4 and 7), suggesting that they are

667 frequently purged by natural selection in the long term. This is indication of a source-sink

668 dynamics, where a pool of conjugative elements (source) is constantly generating variants that

669 require a T4SS in trans that tend to rapidly disappear from populations (sink). Similar processes

670 may explain how plasmids with relaxases become pMOBless.

671 Some transitions from conjugative to mobilizable plasmids have given rise to specialized

672 relaxases that may be advantageous in certain circumstances, as described above. Since most

673 bacterial clades have both conjugative and mobilizable plasmids and half of the genomes with a

674 mobilizable plasmid also contain a conjugative plasmid, there is plenty of opportunity for the

675 novel mobilizable plasmid to meet a conjugative element and transfer to other cells. In such a 
676 context, why should most of the novel pMOBs or pdCONJs be purged by natural selection as

677 suggested by our data? If the relaxase is initially specialized in a T4SS (the one previously

678 encoded in cis), then the novel mobilizable plasmid may have low transfer rates because it

679 requires a very similar T4SS to transfer. This difficulty is amplified by plasmid incompatibility. If

680 the novel mobilizable plasmid keeps its replicon group, then it is incompatible with the most

681 closely related pCONJ. Incompatibility means the two plasmids cannot co-exist in a stable

682 manner and this may decrease the rate of horizontal transmission of the mobilizable plasmid to

683 a point where it will become extinct (if not adaptive to the host). This may explain why

684 observable transitions between conjugative and mobilizable plasmids always involve significant

685 changes in wGRR: plasmids changing the replication machinery have a higher likelihood of

686 surviving the initial stages after the transition. This fits our observation that many novel pMOBs

687 have acquired a different rep type (have a different incompatibility family) and concomitantly

688 endured a drastic change in gene repertoires. Other reasons may contribute to the counter-

689 selection of novel mobilizable plasmids, including lack of coordination in expression of the

690 relaxase and the compatible T4SS encoded in trans, or the existence of remnants of degraded

691 T4SS that can be costly and even toxic to the cell.

692 Transitions in terms of mobility are associated with changes in the classification of replication

693 types of plasmids and the changes in gene repertoires can also affect their classification in

694 terms of PTUs. We show that PTUs frequently contain several mobility types within them. This

695 is consistent with the observation that changes in mobility type are frequent in plasmid

696 evolution, with respect to evolution of the relaxase. Many of these recent changes are not

697 drastic enough to expel a plasmid from a PTU, as shown in figure 9B. However, plasmids that

698 endure substantial changes in their genomes tend to separate from the original PTU. If these

699 transitions are successful, i.e., these plasmids persist and propagate in populations, this may

700 generate novel PTUs, as observed for E5, $F_{S h}$ and E41, which originated from $F_{E}$. These new

701 PTUs may represent specific adaptations of the plasmid genetic structure to the constraints of a

702 new condition, in this case a novel type of mobility. Hence, substantial diversity of genomic

703 content can be found within a PTU, but very substantial diversification of plasmids following

704 changes in mobility often result in novel PTUs.

705 Acknowledgements

706 Eugen Pfeifer for providing the wGRR data and Marie Touchon for providing the ISScan analysis.

707 Manuel Arroyo for comments and suggestions. Jorge Moura de Sousa, Matthieu Haudiquet and

708 Olaya Rendueles-Garcia for the scientific conversations. INCEPTION project [PIA/ANR-16-CONV-

709 0005], Equipe FRM [EQU201903007835], Laboratoire d'Excellence IBEID [ANR-10-LABX-62-

710 IBEID]. PID2020-117923GB-I00 project funded by Spanish Ministry of Science and Innovation to

711 FdIC and MPG-B. 
References

713

714

715

716

717

718

719

720

721

722

723

724

725

726

727

728

729

730

731

732

733

734

735

736

737

738

739

740

741

742

743

744

745

746

747

Abby SS, Neron B, Menager H, Touchon M, Rocha EP. 2014. MacSyFinder: A Program to Mine Genomes for Molecular Systems with an Application to CRISPR-Cas Systems. PLoS One 9:e110726.

Adelberg EA, Pittard J. 1965. Chromosome Transfer in Bacterial Conjugation. Bacteriol Rev 29:161-172.

Bastian M, Heymann S, Jacomy M. 2009. Gephi: an open source software for exploring and manipulating networks. Third international AAAl conference on weblogs and social media.

Blanca-Ordóñez H, Oliva-García JJ, Pérez-Mendoza D, Soto MJ, Olivares J, Sanjuán J, Nogales J. 2010. pSymA-dependent mobilization of the Sinorhizobium meliloti pSymB megaplasmid. Journal of bacteriology 192:6309-6312.

Blesa A, Baquedano I, Quintáns NG, Mata CP, Castón JR, Berenguer J. 2017. The transjugation machinery of Thermus thermophilus: Identification of TdtA, an ATPase involved in DNA donation. PLoS Genet 13:e1006669.

Cabezón E, Lanka E, de la Cruz F. 1994. Requirements for mobilization of plasmids RSF1010 and ColE1 by the IncW plasmid R388: trwB and RP4 traG are interchangeable. J Bacteriol 176:4455-4458.

Cabezón E, Sastre JI, de la Cruz F. 1997. Genetic evidence of a coupling role for the TraG protein family in bacterial conjugation. Mol Gen Genet 254:400-406.

Carattoli A. 2013. Plasmids and the spread of resistance. International Journal of Medical Microbiology 303:298-304.

Carattoli A, Zankari E, García-Fernández A, Voldby Larsen M, Lund O, Villa L, Møller Aarestrup F, Hasman H. 2014. <em>In Silico</em> Detection and Typing of Plasmids using PlasmidFinder and Plasmid Multilocus Sequence Typing. Antimicrobial Agents and Chemotherapy 58:3895-3903.

Cerveau N, Leclercq S, Bouchon D, Cordaux R. 2011. Evolutionary dynamics and genomic impact of prokaryote transposable elements. Evolutionary biology-concepts, biodiversity, macroevolution and genome evolution: Springer. p. 291-312.

Che Y, Yang Y, Xu X, Břinda K, Polz MF, Hanage WP, Zhang T. 2021. Conjugative plasmids interact with insertion sequences to shape the horizontal transfer of antimicrobial resistance genes. Proc Natl Acad Sci U S A 118:e2008731118.

Coluzzi C, Guédon G, Devignes M-D, Ambroset C, Loux V, Lacroix T, Payot S, Leblond-Bourget N. 2017. A glimpse into the world of integrative and mobilizable elements in streptococci reveals an unexpected diversity and novel families of mobilization proteins. Frontiers in microbiology 8:443. 
765

766

767

768

769

770

771

772

773

774

775

776

777

778

779

780

781

782

Cury J, Oliveira PH, de la Cruz F, Rocha EPC. 2018. Host Range and Genetic Plasticity Explain the Coexistence of Integrative and Extrachromosomal Mobile Genetic Elements. Mol Biol Evol 35:2230-2239.

Cury J, Touchon M, Rocha EP. 2017. Integrative and conjugative elements and their hosts: composition, distribution and organization. Nucl Acid Res 45:8943-8956.

de la Cruz F, Davies J. 2000. Horizontal gene transfer and the origin of species: lessons from bacteria. Trends Microbiol 8:128-133.

de la Cruz F, Frost LS, Meyer RJ, Zechner E. 2010. Conjugative DNA Metabolism in Gramnegative Bacteria. FEMS Microbiol Rev 34:18-40.

Denise R, Abby SS, Rocha EP. 2019. Diversification of the type IV filament superfamily into machines for adhesion, protein secretion, DNA uptake, and motility. PLoS Biol 17:e3000390.

Eberhard WG. 1990. Evolution in bacterial plasmids and levels of selection. Q Rev Biol 65:3-22.

Eddy SR. 2011. Accelerated Profile HMM Searches. PLoS Comput Biol 7:e1002195.

Fernandez-Lopez R, de Toro M, Moncalian G, Garcillan-Barcia MP, de la Cruz F. 2016. Comparative Genomics of the Conjugation Region of F-like Plasmids: Five Shades of F. Front Mol Biosci 3:71.

Fürste JP, Pansegrau W, Ziegelin G, Kröger M, Lanka E. 1989. Conjugative transfer of promiscuous IncP plasmids: interaction of plasmid-encoded products with the transfer origin. Proc Natl Acad Sci U S A 86:1771-1775.

Garcillan-Barcia MP, Cuartas-Lanza R, Cuevas A, de la Cruz F. 2019. Cis-Acting Relaxases Guarantee Independent Mobilization of MOBQ4 Plasmids. Frontiers in Microbiology 10:2557.

Garcillan-Barcia MP, Francia MV, de la Cruz F. 2009. The diversity of conjugative relaxases and its application in plasmid classification. FEMS Microbiol Rev 33:657-687.

Garcillan-Barcia MP, Redondo-Salvo S, Vielva L, de la Cruz F. 2020. MOBscan: Automated Annotation of MOB Relaxases. Methods Mol Biol 2075:295-308.

Geng P, Cheng J, Yuan Z, Xiong H, Wang H, Hu X. 2021. Horizontal transfer of large plasmid with type IV secretion system and mosquitocidal genomic island with excision and integration capabilities in Lysinibacillus sphaericus. Environ Microbiol 23:5131-5146.

Gonzalez-Perez B, Lucas M, Cooke LA, Vyle JS, de la Cruz F, Moncalián G. 2007. Analysis of DNA processing reactions in bacterial conjugation by using suicide oligonucleotides. Embo $j$ 26:3847-3857.

Guglielmini J, de la Cruz F, Rocha EPC. 2013. Evolution of Conjugation and Type IV Secretion Systems. Mol Biol Evol 30:315-331. 
Guglielmini J, Quintais L, Pilar Garcillan-Barcia M, de la Cruz F, Rocha EPC. 2011. The Repertoire of ICE in Prokaryotes Underscores the Unity, Diversity, and Ubiquity of Conjugation. Plos Genet 7: e1002222.

Guy L, Kultima JR, Andersson SG. 2010. genoPlotR: comparative gene and genome visualization in R. Bioinformatics 26:2334-2335.

Hall JPJ, Brockhurst MA, Harrison E. 2017. Sampling the mobile gene pool: innovation via horizontal gene transfer in bacteria. Philos Trans R Soc Lond B Biol Sci 372.

Harrison PW, Lower RP, Kim NK, Young JP. 2010. Introducing the bacterial 'chromid': not a chromosome, not a plasmid. Trends Microbiol 18:141-148.

Hauser M, Mayer CE, Söding J. 2013. kClust: fast and sensitive clustering of large protein sequence databases. BMC Bioinformatics 14:248.

Hauser M, Steinegger M, Soding J. 2016. MMseqs software suite for fast and deep clustering and searching of large protein sequence sets. Bioinformatics 32:1323-1330.

Humphrey S, San Millán Á, Toll-Riera M, Connolly J, Flor-Duro A, Chen J, Ubeda C, MacLean RC, Penadés JR. 2021. Staphylococcal phages and pathogenicity islands drive plasmid evolution. Nature Communications 12:5845.

Ishikawa SA, Zhukova A, Iwasaki W, Gascuel O. 2019. A Fast Likelihood Method to Reconstruct and Visualize Ancestral Scenarios. Mol Biol Evol 36:2069-2085.

Johnson CM, Grossman AD. 2015. Integrative and conjugative elements (ICEs): what they do and how they work. Annual review of genetics 49:577-601.

Johnson TJ, Nolan LK. 2009. Pathogenomics of the virulence plasmids of Escherichia coli. Microbiology and Molecular Biology Reviews 73:750-774.

Katoh K, Standley DM. 2014. MAFFT: iterative refinement and additional methods. Methods Mol Biol 1079:131-146.

Klümper U, Droumpali A, Dechesne A, Smets BF. 2014. Novel assay to measure the plasmid mobilizing potential of mixed microbial communities. Frontiers in microbiology 5:730.

Kobayashi H. 2018. Regeneration of Escherichia coli from Minicells through Lateral Gene Transfer. J Bacteriol 200:e00630-00617.

Koskiniemi S, Sun S, Berg OG, Andersson DI. 2012. Selection-Driven Gene Loss in Bacteria. PLOS Genetics 8:e1002787.

Lacroix B, Citovsky V. 2018. Beyond Agrobacterium-mediated transformation: horizontal gene transfer from bacteria to eukaryotes. Curr Topics Microbiol Immunol 418:443-462.

Lanza VF, Baquero F, de la Cruz F, Coque TM. 2017. AcCNET (Accessory Genome Constellation Network): comparative genomics software for accessory genome analysis using bipartite networks. Bioinformatics 33:283-285.

Liu MA, Kwong SM, Jensen SO, Brzoska AJ, Firth N. 2013. Biology of the staphylococcal conjugative multiresistance plasmid pSK41. Plasmid 70:42-51. 
Lorenzo-Díaz F, Fernández-López C, Garcillán-Barcia MP, Espinosa M. 2014. Bringing them together: plasmid pMV158 rolling circle replication and conjugation under an evolutionary perspective. Plasmid 74:15-31.

Meyer R. 2009. Replication and conjugative mobilization of broad host-range IncQ plasmids. Plasmid 62:57-70.

Mira A, Ochman H, Moran NA. 2001. Deletional bias and the evolution of bacterial genomes. Trends Genet 17:589-596.

Nguyen LT, Schmidt HA, von Haeseler A, Minh BQ. 2015. IQ-TREE: a fast and effective stochastic algorithm for estimating maximum-likelihood phylogenies. Mol Biol Evol 32:268-274.

Nuti M, Lepidi A, Prakash R, Schilperoort R, Cannon F. 1979. Evidence for nitrogen fixation (nif) genes on indigenous Rhizobium plasmids. Nature 282:533-535.

O'Brien FG, Yui Eto K, Murphy Riley JT, Fairhurst Heather M, Coombs GW, Grubb WB, Ramsay JP. 2015. Origin-of-transfer sequences facilitate mobilisation of non-conjugative antimicrobial-resistance plasmids in Staphylococcus aureus. Nucleic Acids Research 43:7971-7983.

Paradis E, Schliep K. 2019. ape 5.0: an environment for modern phylogenetics and evolutionary analyses in R. Bioinformatics 35:526-528.

Partridge SR, Kwong SM, Firth N, Jensen SO. 2018. Mobile Genetic Elements Associated with Antimicrobial Resistance. Clin Microbiol Rev 31:e00088-00017.

Pedersen T, Sekyere JO, Govinden U, Moodley K, Sivertsen A, Samuelsen $\varnothing$, Essack SY, Sundsfjord A. 2018. Spread of Plasmid-Encoded NDM-1 and GES-5 Carbapenemases among Extensively Drug-Resistant and Pandrug-Resistant Clinical Enterobacteriaceae in Durban, South Africa. Antimicrob Agents Chemother 62:e02178-02117.

Perez-Mendoza D, Lucas M, Munoz S, Herrera-Cervera JA, Olivares J, de la Cruz F, Sanjuan J. 2006. The relaxase of the Rhizobium etli symbiotic plasmid shows nic site cis-acting preference. J Bacteriol 188:7488-7499.

Ramachandran G, Miguel-Arribas A, Abia D, Singh PK, Crespo I, Gago-Cordoba C, Hao JA, LuqueOrtega JR, Alfonso C, Wu L. 2017. Discovery of a new family of relaxases in Firmicutes bacteria. PLoS genetics 13:e1006586.

Ramsay JP, Firth N. 2017. Diverse mobilization strategies facilitate transfer of non-conjugative mobile genetic elements. Current opinion in microbiology 38:1-9.

Ramsay JP, Kwong SM, Murphy RJ, Yui Eto K, Price KJ, Nguyen QT, O'Brien FG, Grubb WB, Coombs GW, Firth N. 2016. An updated view of plasmid conjugation and mobilization in Staphylococcus. Mobile genetic elements 6:e1208317.

Rankin DJ, Rocha EPC, Brown SP. 2011. What traits are carried on mobile genetic elements, and why? Heredity 104:1-10.

Redondo-Salvo S, Bartomeus-Peñalver R, Vielva L, Tagg KA, Webb HE, Fernández-López R, de la Cruz F. 2021. COPLA, a taxonomic classifier of plasmids. BMC Bioinformatics 22:390. 
858

859

860

861

862

863

864

865

866

867

868

869

870

871

872

873

874

875

876

877

878

879

880

881

882

883

884

885 886

887

888

889

890

891

892

893

Redondo-Salvo S, Fernández-López R, Ruiz R, Vielva L, de Toro M, Rocha EP, Garcillán-Barcia $M P$, de la Cruz F. 2020. Pathways for horizontal gene transfer in bacteria revealed by a global map of their plasmids. Nature communications 11:1-13.

Revilla C, Garcillán-Barcia MP, Fernández-López R, Thomson NR, Sanders M, Cheung M, Thomas $\mathrm{CM}$, de la Cruz F. 2008. Different pathways to acquiring resistance genes illustrated by the recent evolution of IncW plasmids. Antimicrob Agents Chemother 52:1472-1480.

San Millan A, Craig MacLean R. 2019. Fitness costs of plasmids: a limit to plasmid transmission. Microbiol Spectr 5:65-79.

Sastre JI, Cabezón E, de la Cruz F. 1998. The carboxyl terminus of protein TraD adds specificity and efficiency to F-plasmid conjugative transfer. J Bacteriol 180:6039-6042.

Smillie C, Pilar Garcillan-Barcia M, Victoria Francia M, Rocha EPC, de la Cruz F. 2010. Mobility of Plasmids. Microbiol Mol Biol Rev 74:434-452.

Soler N, Robert E, Chauvot de Beauchêne I, Monteiro P, Libante V, Maigret B, Staub J, Ritchie DW, Guédon G, Payot S, et al. 2019. Characterization of a relaxase belonging to the $\mathrm{MOB}(\mathrm{T})$ family, a widespread family in Firmicutes mediating the transfer of ICEs. Mob DNA 10:18.

Soucy SM, Huang J, Gogarten JP. 2015. Horizontal gene transfer: building the web of life. Nature Reviews Genetics 16:472.

Stalder T, Press MO, Sullivan S, Liachko I, Top EM. 2019. Linking the resistome and plasmidome to the microbiome. Isme j 13:2437-2446.

Steenwyk JL, Buida III TJ, Li Y, Shen X-X, Rokas A. 2020. ClipKIT: A multiple sequence alignment trimming software for accurate phylogenomic inference. PLoS biology 18:e3001007.

Steinegger M, Meier M, Mirdita M, Vohringer H, Haunsberger SJ, Soding J. 2019. HH-suite3 for fast remote homology detection and deep protein annotation. BMC Bioinformatics 20:473.

Szabó M, Murányi G, Kiss J. 2021. IncC helper dependent plasmid-like replication of Salmonella Genomic Island 1. Nucleic acids research 49:832-846.

Trieu-Cuot P, Carlier C, Martin P, Courvalin P. 1987. Plasmid transfer by conjugation from Escherichia coli to Gram-positive bacteria. FEMS Microbiology Letters 48:289-294.

Waskom ML. 2021. Seaborn: statistical data visualization. Journal of Open Source Software 6:3021.

Yui Eto K, Kwong SM, LaBreck PT, Crow JE, Traore DAK, Parahitiyawa N, Fairhurst HM, Merrell DS, Firth N, Bond CS, et al. 2021. Evolving origin-of-transfer sequences on staphylococcal conjugative and mobilizable plasmids-who's mimicking whom? Nucleic Acids Res 49:5177-5188. 